\title{
Potent inhibition of HIV-1 by TRIM5-cyclophilin fusion proteins engineered from human components
}

\author{
Martha R. Neagu,1,2,3 Patrick Ziegler, ${ }^{2}$ Thomas Pertel,2,3 Caterina Strambio-De-Castillia, ${ }^{2}$ \\ Christian Grütter, ${ }^{4}$ Gladys Martinetti, ${ }^{5}$ Luca Mazzucchelli, ${ }^{6}$ Markus Grütter, ${ }^{4}$ \\ Markus G. Manz, 2,7 and Jeremy Luban'1,2,3,8

\begin{abstract}
${ }^{1}$ Department of Microbiology and Columbia University, New York, New York, USA. ${ }^{2}$ Institute for Research in Biomedicine, Bellinzona, Switzerland. ${ }^{3}$ Department of Microbiology and Molecular Medicine, University of Geneva, Geneva, Switzerland. ${ }^{4}$ Department of Biochemistry, University of Zurich, Zurich, Switzerland. ${ }^{5}$ Institute of Microbiology, Bellinzona, Switzerland. ${ }^{6}$ Institute for Pathology, Locarno, Switzerland. ${ }^{7}$ Department of Internal Medicine, University Hospital Zurich, Zurich, Switzerland. ${ }^{8}$ Department of Medicine, Columbia University, New York, New York, USA.
\end{abstract}

\begin{abstract}
New World monkeys of the genus Aotus synthesize a fusion protein (AoT5Cyp) containing tripartite motif-containing 5 (TRIM5) and cyclophilin A (CypA) that potently blocks HIV-1 infection. We attempted to generate a human HIV-1 inhibitor modeled after AoT5Cyp, by fusing human CypA to human TRIM5 (hT5Cyp). Of 13 constructs, 3 showed substantial HIV-1-inhibitory activity when expressed in human cell lines. This activity required capsid binding by CypA and correlated with CypA linkage to the TRIM5a capsid-specificity determinant and the ability to form cytoplasmic bodies. CXCR4- and CCR5-tropic HIV-1 clones and primary isolates were inhibited from infecting multiple human macrophage and $T$ cell lines and primary cells by hT5Cyp, as were HIV-2 ${ }_{\mathrm{ROD}}$, SIV $\mathrm{AGM}_{\text {tan, FIV }}$ PET, and a circulating HIV-1 isolate previously reported to be AoT5Cyp resistant. The anti-HIV-1 activity of hT5Cyp was surprisingly more effective than that of the well-characterized rhesus TRIM5 $\alpha$, especially in T cells. hT5Cyp also blocked HIV-1 infection of primary CD4 $4^{+} \mathrm{T}$ cells and macrophages and conferred a survival advantage to these cells without disrupting their function. Extensive attempts to elicit

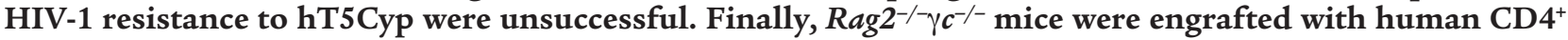
T cells that had been transduced by optimized lentiviral vectors bearing hT5Cyp. Upon challenge with HIV-1, these mice showed decreased viremia and productive infection in lymphoid organs and preserved numbers of human $\mathrm{CD}^{+} \mathrm{T}$ cells. We conclude that hT5Cyp is an extraordinarily robust inhibitor of HIV-1 replication and a promising anti-HIV-1 gene therapy candidate.
\end{abstract}

\section{Introduction}

Over 60 million people have been infected with HIV-1, and nearly half of these people have died as a consequence of this infection (1). Despite the discovery 25 years ago of HIV-1, it continues to kill several million people each year. In theory, the clearest path to the elimination of the HIV-1 pandemic would be an effective antiHIV-1 vaccine. In practice, the virus has proven to be an elusive target for the immune system $(2,3)$. Combination antiviral therapy potently suppresses HIV-1 replication and resultant disease, but these treatments are plagued with complications and they do not eliminate the virus.

The difficulties of controlling HIV-1 have stimulated thinking about alternatives to conventional vaccination or life-long pharmacotherapy. Among these creative possibilities are anti-HIV-1 gene therapy $(4,5)$. Ideally, gene therapy should potently suppress HIV-1 replication without eliciting viral resistance. While all steps of the viral life cycle are potential gene therapy targets, blocking the virus before RT would preclude the genetic diversity that permits emergence of viral resistance. Additionally, targeting the virus before HIV-1 cDNA is ligated into host chromosomal DNA would prevent the virus from becoming a heritable genetic element in that cellular lineage. The discovery that certain tripartite motif-containing 5 (TRIM5) orthologs inhibit HIV-1 infection immediately after

Conflict of interest: The authors have declared that no conflict of interest exists. Citation for this article: J. Clin. Invest. 119:3035-3047 (2009). doi:10.1172/JCI39354. the virus enters otherwise susceptible cells (6-9) raised the prospect that these host factors might be exploited in HIV-1 gene therapy.

The $\alpha$ isoform of TRIM5 (TRIM5 $\alpha$ ) contains a C-terminal PRYSPRY domain that is required for TRIM5 $\alpha$ binding to the capsid (CA) of restriction-sensitive retroviruses $(10,11)$. The specificity of the PRYSPRY-CA interaction determines which retrovirus a given TRIM5 $\alpha$ ortholog inhibits (12). While human TRIM5 $\alpha$ (hTRIM5 $\alpha$ ) weakly blocks HIV-1, it potently blocks N-tropic murine leukemia virus (N-MLV). In contrast, rhesus TRIM5 $\alpha$ (rhTRIM5 $\alpha$ ) inhibits HIV-1, but only weakly inhibits N-MLV. The specificity of retroviral restriction and the modular nature of the TRIM5 components are further demonstrated by the enhanced HIV-1 restriction activity that results when the hTRIM5 $\alpha$ PRYSPRY domain is replaced with that from rhTRIM5 $\alpha$ (13-16). Furthermore, anti-HIV-1 activity is observed when other TRIM family members (17) or even the murine antiretroviral factor Fv1 (18) are fused to an HIV-1 CA-binding protein.

The TRIM5 gene in the New World owl monkey (genus Aotus) is unusual. It resulted from retrotransposition of the cyclophilin A (СурA) cDNA into intron $7(7,19)$. CypA is a CA-binding protein (20), and the Aotus T5Cyp fusion (AoT5Cyp) prevents HIV-1 infection $(7,19)$. AoT5Cyp has several properties that make it appealing for gene therapy. It potently inhibits HIV-1 infection when expressed in human cells $(7,19)$, acting on the virus within minutes of entry $(8,9)$. Furthermore, AoT5Cyp is the only TRIM5 allele in 10 Aotus species (21), indicating that cells bearing AoT5Cyp retain 
A

MASGILVNVKEEVTCPICLELLTQPLSLDCGHSFCQACLTANHKKSMLDK

GESSCPVCRISYQPENIRPNRHVANLVEKLREVKLSPEGQKVDHCARHGE

$B$ Box 2

efgabcdefgabcdefgab

KLLLFCQEDGKVICWLCERSQEHRGHHTFPTEEVAQEYQVKLQAALEMLR

cdefgabcdefgabcdefg.

QKQQEAEELEADIREEKASWKTQIQYDKTNVLADFEQLRDILDWEESNEL

gabcdefgabcdefgabcdefgabcdefgab M244

QNLEKEEEDILKSLTNSETEMVQQTQSLRELISDLEHRLQGSVMELLQGV

M284 W298

DGVIKRTENVTLKKPETFPKNQRRVFRAPDLKGMLEVFRELTDVRRYWVD

$\begin{array}{lllll}\text { T302 } & \text { S309 } & \text { S3.14 } & \text { S322 } & \text { A331 }\end{array}$

VTVAPNNISCAVISEDKRQVSSPKPQIIYGARGTRYQTFVNFNYCTGILG

$\begin{array}{lll}\text { G357 T369 G398 } & \end{array}$

SQSITSGKHYWEVDVSKKTAWILGVCAGFQPDAMCNIEKNENYQPKYGYW

VIGLEEGVKCSAFQDSSFHTPSVPFIVPLSVIICPDRVGVFLDYEACTVS

FFNITNHGFLIYKFSHCSFSQPVFPYLNPRKCGVPMTLCSPSS
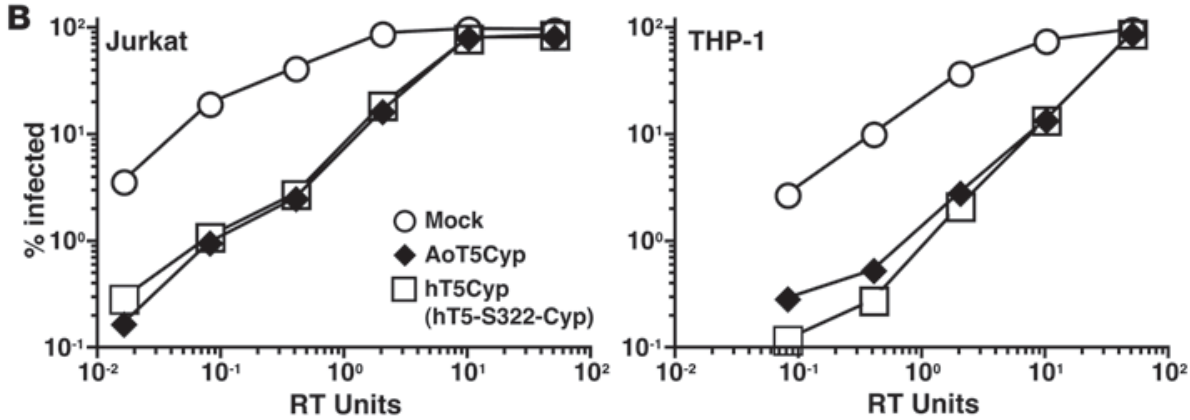

\section{Figure 1}

Identification of human T5Cyp proteins that potently restrict HIV-1. (A) The sequence of hTRIM $5 \alpha$ protein. Lowercase letters indicate the register of the predicted coiled coils. Boxed areas indicate PRYSPRY residues. Vertical lines indicate the site of fusion of CypA to TRIM5. Numbers indicate the TRIM5 amino acid to which CypA is fused, and are color coded for restriction phenotype (red, potently restrictive; orange, variably restrictive; green, permissive). (B) Jurkat T cells and THP- 1 monocytes were transduced with vectors encoding puromycin resistance and either AoT5Cyp or hT5Cyp (hT5-S222-Cyp) fusion proteins. Pools of puromycin-resistant cells were infected with increasing amounts of a single-cycle HIV-1 vector encoding GFP (measured as RT units). The percentage of GFP+ cells was determined 48 hours later. functionality. One drawback of AoT5Cyp is that it is not a human protein, and if employed as gene therapy, it might elicit an immune response (22). Here we engineered hT5Cyp fusion proteins exhibiting HIV-1 restriction activity comparable to AoT5Cyp and possessing all the properties desired of anti-HIV-1 gene therapy.

\section{Results}

Design of hT5Cyp fusions with potent anti-HIV-1 activity. Our goal was to generate a hT5Cyp fusion protein modeled after AoT5Cyp using only human components. A cryptic splice acceptor at the AoT5Cyp fusion junction results in the synthesis of 12 amino acids derived from the CypA 5'UTR (7). An equivalent human fusion cannot be engineered due to lack of sequence homology in the CypA 5'UTR. Sequences from TRIM5 $\gamma$ and TRIM5 , of similar length and character to the AoT5Cyp 5'UTR, were included at the TRIM5-CypA junction, but these failed to result in proteins with anti-HIV-1 activity. hCypA was then fused directly to hTRIM5 $\alpha$ at 11 different positions along its linear sequence, eliminating subsequent hTRIM5 $\alpha$ sequences and resulting in hT5Cyp fusions consisting entirely of components expressed in human cells (Figure 1A). Each hT5Cyp fusion was engineered into a bicistronic lentiviral vector (FUPI; Supplemental Figure 1A; supplemental material available online with this article; doi:10.1172/JCI39354DS1). Vector stocks generated for each construct were shown to have equivalent titers and then used to generate stable cell lines.

Pools of Jurkat or THP-1 cells, each bearing a different hT5Cyp construct, were tested for restriction activity against a single-cycle HIV-1-GFP vector. Among the hT5Cyp constructs, 3 had activity comparable to AoT5Cyp (see representative data in Figure 1B for hT5-S322-Cyp). This fusion was used in all subsequent experiments and will be referred to as "hT5Cyp". Data for all of the fusion constructs is shown in Supplemental Figure 1. hT5Cyp also restricted HIV-1 in promonocytic U937 cells, the CEM-SS, SUPT1, and H9 T cell lines, and the 293T embryonic kidney cell line (data not shown). Like AoT5Cyp (9), hT5Cyp blocked viral cDNA accumulation after acute infection, as measured by quantitative PCR (Supplemental Figure 1B).

Structural requirements for $b T 5 C y p$-mediated HIV-1 restriction activity. Despite the apparent modularity of TRIM5 (13-16), most hT5Cyp fusions lacked anti-HIV-1 activity. Steady-state protein levels in stably transduced Jurkat $\mathrm{T}$ cells did not correlate with restriction activity - AoT5Cyp and restrictive hT5Cyp fusions were undetectable by immunoblot, while some inactive mutants were highly expressed. To increase the possibility of detection, hT5Cyp proteins were expressed as triple-FLAG-tagged fusions by plasmid transfection of $293 \mathrm{~T}$ cells. Anti-FLAG immunoprecipitates were probed in immunoblots with anti-CypA antibody. Two fusions with no detectable restriction activity, hT5-M244-Cyp and hT5T302-Cyp, were expressed at high levels, whereas fusions with potent anti-HIV-1 activity were variably expressed at moderate to high levels (hT5-S309-Cyp and hT5-S322-Cyp) or at low levels (hT5-A331-Cyp and AoT5Cyp) (Figure 2A). Again, restriction activity did not correlate with protein levels.

Lack of activity could result from failure to bind HIV-1 CA. Glutathione S transferase-CA (GST-CA) fusion protein was used to test CA-binding activity of hT5Cyp proteins produced in 293T cells. Among hT5Cyp fusions with no anti-HIV-1 activity, hT5-T302-Cyp bound HIV-1 CA as well as the potently restrictive hT5-S322-Cyp (Fig- 
A

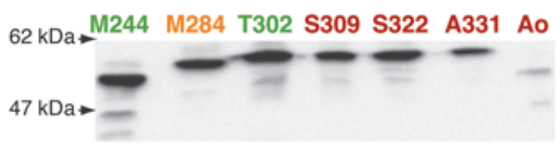

B

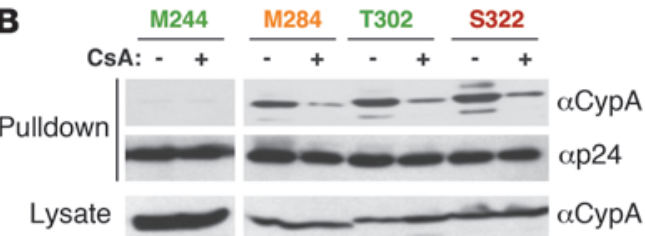

C
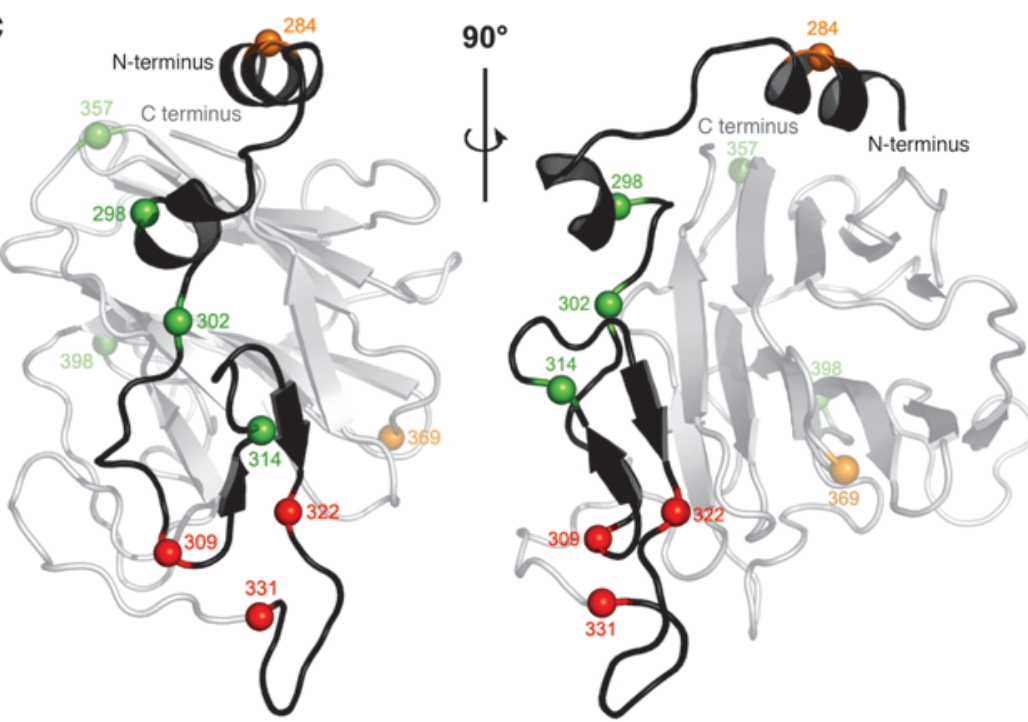

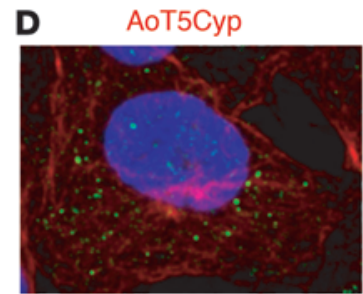

309

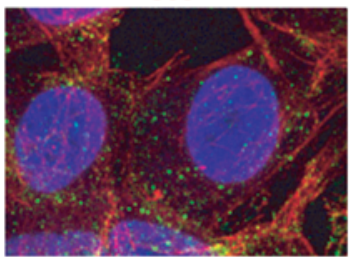

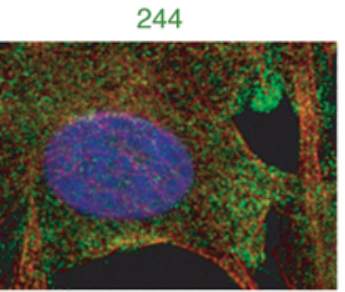

322

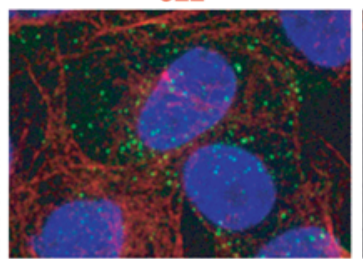

284

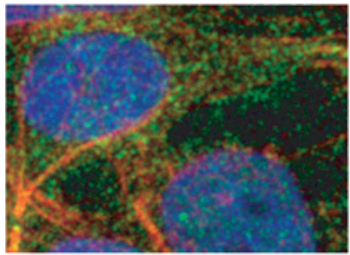

331

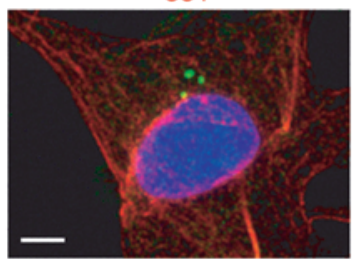

302

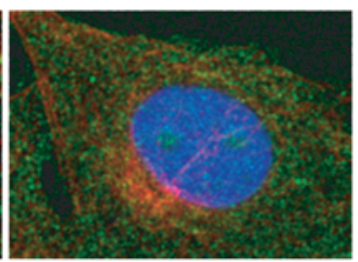

\section{.}


A
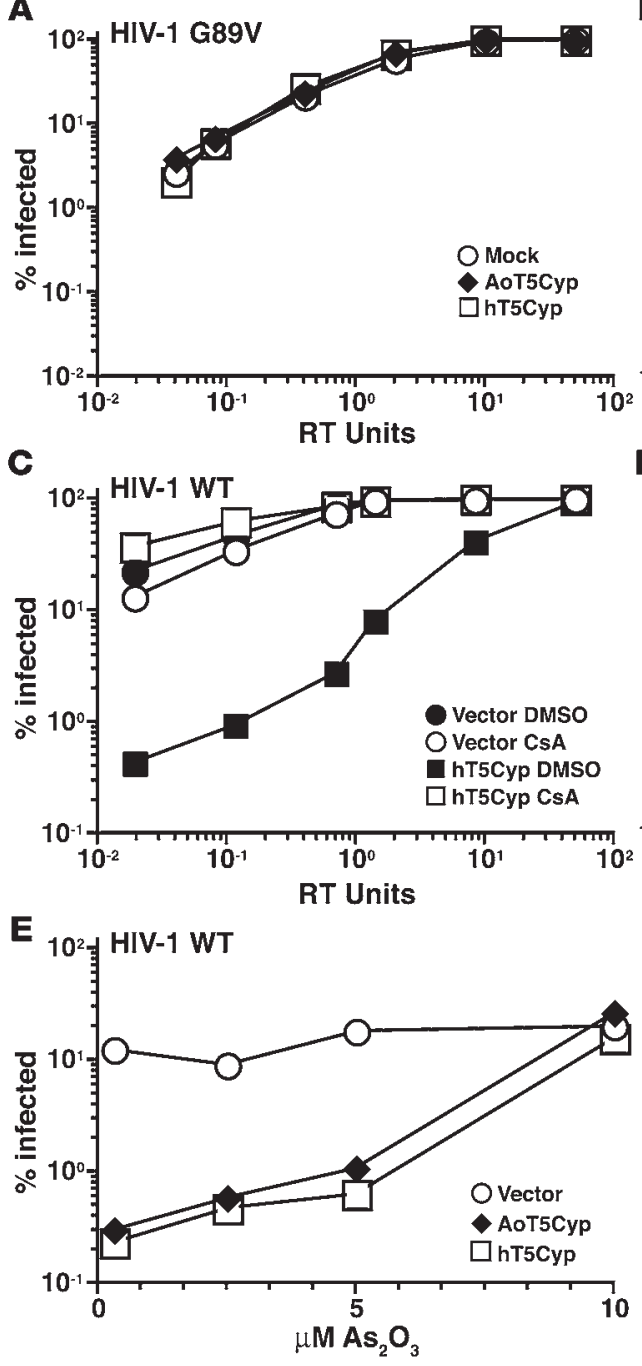
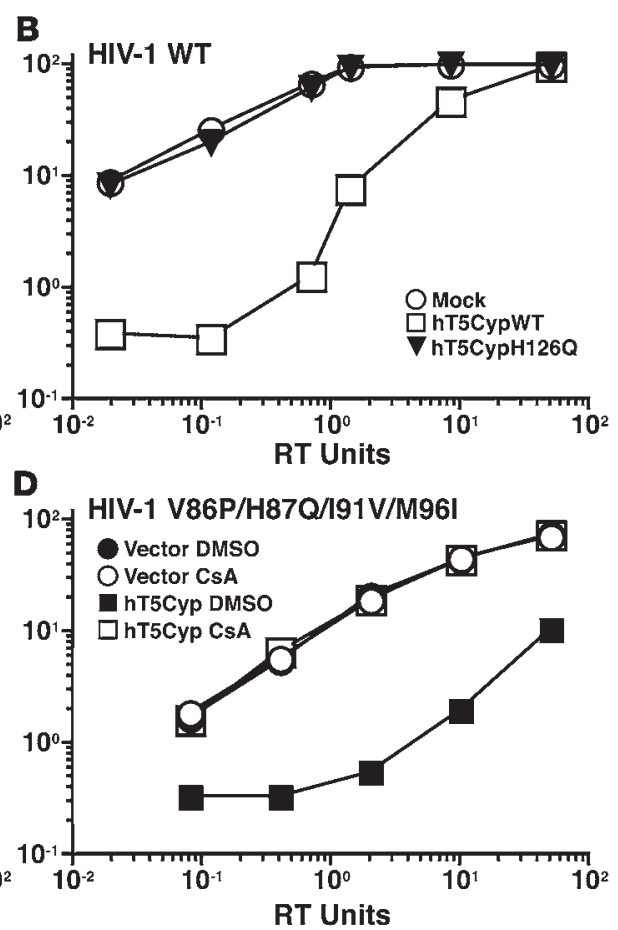

Figure 3

TRIM5 and CypA components each make essential contributions to T5Cyp restriction activity. (A-C) Disruption of the CA-CypA interaction by the G89V mutation in HIV-1 CA (A), the H126Q mutation in CypA (B), or the competitive inhibitor CsA at $2.5 \mu \mathrm{M}$ (C) blocked HIV-1 restriction activity in CRFK cells stably expressing the indicated TRIM5 proteins or controls. Cells were challenged with increasing amounts of HIV-1-GFP single-cycle vector bearing the G89V mutation in CA (A) or wild-type HIV-1 GFP vector (B and C). (D) Introduction of a naturally occurring quadruple mutation flanking the CA-CypA binding site in HIV-1 CA did not abrogate hT5Cyp-mediated restriction of HIV-1. CRFK cells stably expressing T5Cyp or control were infected with increasing amounts of an HIV-1 GFP vector containing the V86P/H87Q/I91V/M96I mutation in CA. (E) Disruption of TRIM5 by $\mathrm{As}_{2} \mathrm{O}_{3}$ blocked HIV-1 restriction activity by T5Cyp. CRFK cells stably expressing the indicated T5Cyp were infected with HIV-1 GFP vector in the presence of increasing amounts of $\mathrm{As}_{2} \mathrm{O}_{3}$. In each case, the percentage of GFP+ cells was measured by flow cytometry 48 hours after infection. tion surface $(23,24)$ (Figure $2 \mathrm{C})$, the main specificity determinant for HIV-1 restriction activity $(15,16,25)$. No inactive hT5Cyp proteins mapped to this region (Figure $2 \mathrm{C}$ ). Indirect immunofluorescence showed that AoT5Cyp and all 3 hT5Cyps with potent antiHIV-1 activity formed distinct cytoplasmic bodies (Figure 2D). In contrast, diffuse cytoplasmic staining was observed for hT5Cyp fusions that lacked restriction activity. These results suggest that there are conformational requirements for antiviral activity, determined by the site of CypA fusion and revealed by the ability to form cytoplasmic bodies.

bT5Cyp restriction activity depends on both the hCypA and hTRIM5 components. CypA is an abundant protein with potential to inhibit hT5Cyp activity by competing for CA binding. The magnitude restriction of a single-cycle HIV-1-GFP vector by hT5Cyp was unaffected by CypA (Supplemental Figure 2D), as determined in Jurkat $\mathrm{T}$ cells with a stable knockdown of endogenous CypA (26). To confirm that hT5Cyp proteins have the same CA-binding requirements as AoT5Cyp, stable hT5Cyp lines were generated using CRFK, a feline fibroblast that lacks endogenous TRIM5. These cells were challenged with a single-cycle HIV-1-GFP vector containing a mutation in CA (G89V) that disrupts interaction with CypA (7). As expected, HIV-1-G89V was less fit than wild-type
HIV-1, but neither AoT5Cyp nor hT5Cyp restricted this virus (Figure 3A). The CA-CypA interaction is also disrupted by the CypAH126Q mutation (27), and hT5Cyp bearing the H126Q mutation in its CypA domain had no detectable anti-HIV-1 restriction activity (Figure 3B). CsA, a competitive inhibitor of the CypA-CA interaction (20), abrogated HIV-1 restriction by AoT5Cyp and hT5Cyp (Figure 3C). HIV-1-V86P/H87Q/I91V/M96I, a CA variant circulating in humans infected with HIV-1, is reported to be resistant to CsA and to AoT5Cyp-mediated HIV-1 restriction (28). Though HIV-1-V86P/H87Q/I91V/M96I was indeed resistant to CsA, hT5Cyp blocked infection by single-cycle vectors bearing this CA variation (Figure 3D).

To assess the contribution of the TRIM5 domain to T5Cypmediated restriction, CRFK cell lines expressing AoT5Cyp or hT5Cyp were treated with arsenic trioxide $\left(\mathrm{As}_{2} \mathrm{O}_{3}\right)$. This drug was previously shown to inhibit TRIM5-mediated restriction (29). AoT5Cyp and hT5Cyp restriction activity was blocked to the same extent by $\mathrm{As}_{2} \mathrm{O}_{3}$ (Figure 3E).

AoT5Cyp restricts $\mathrm{HIV}-1_{\mathrm{NL} 4-3}, \mathrm{HIV}-2_{\mathrm{ROD}}, \mathrm{SIV}_{\mathrm{AGM}}$ tan, and FIV $\mathrm{PET}_{\mathrm{PET}}$, all viruses encoding a CA that binds CypA (7, 9, 19, 20, 30-32). АоТ5Сур and hT5Cyp restricted single-cycle GFP vectors derived from all 4 of these lentiviruses (Figure 4, A-D). CypA does not bind to $\operatorname{SIV}_{\mathrm{MAC}} 251$ 
A

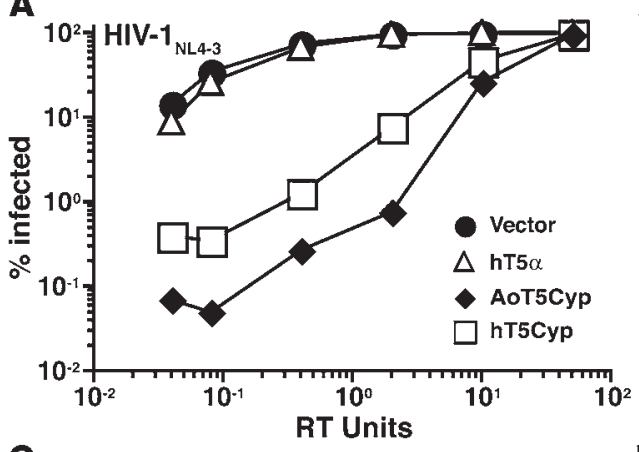

C

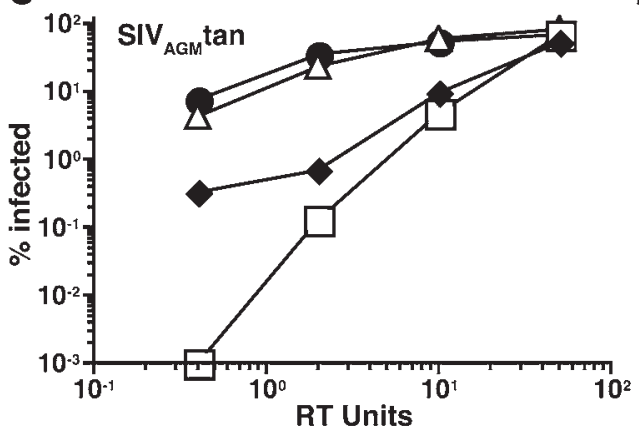

E

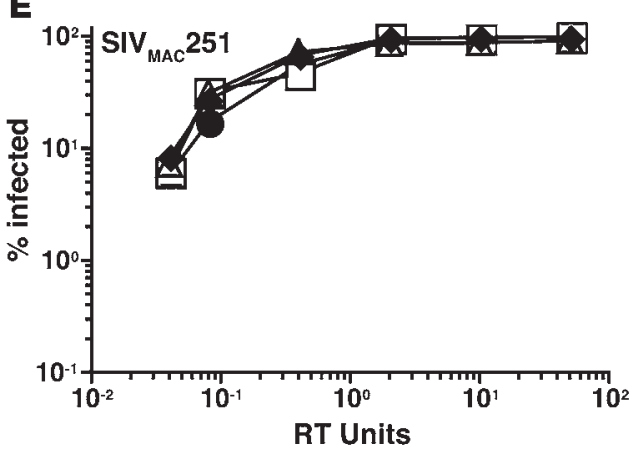

B

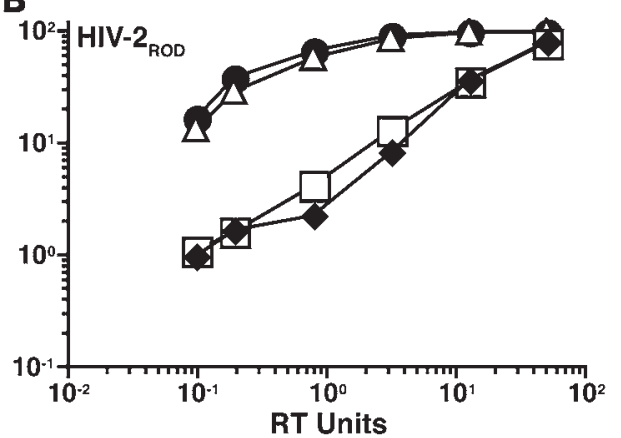

D

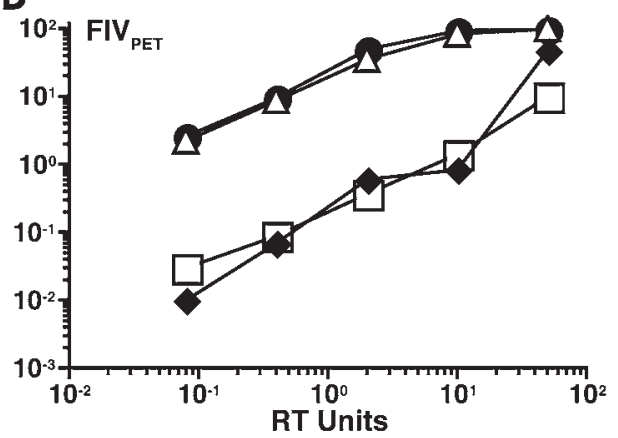

Figure 4

T5Cyp potently restricts lentiviruses that encode CA with CypA-binding activity. (A-E) CRFK cells stably expressing the indicated TRIM5 proteins were transduced with increasing amounts of single-cycle GFP vectors derived from $\mathrm{HIV}-1_{\text {NL4-3 }}(\mathbf{A})$, HIV-2 ROD (B), SIV AGMtan (C), FIV $_{\text {PET }}$

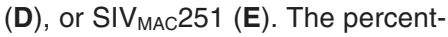
age of GFP+ cells was determined 48 hours later.
CA, and neither AoT5Cyp nor hT5Cyp restricted a SIV $\mathrm{MAC}_{251 \mathrm{GFP}}$ vector (Figure $4 \mathrm{E}$ ). These results show that, with respect to CA recognition by the CypA domain, and effector function by the TRIM5 domain, AoT5Cyp and hT5Cyp behave similarly.

bT5Cyp is more potent than rhTRIM5 $\alpha$. In contrast to hTRIM5 $\alpha$, the TRIM $5 \alpha$ ortholog from rhesus monkeys (rhTRIM5 $\alpha$ ) potently restricts HIV-1 (6). Chimeric proteins in which critical residues from the rhTRIM5 $\alpha$ PRYSPRY domain replace corresponding residues in the human ortholog exhibit HIV-1 restriction activity approaching that of rhTRIM5 $\alpha(14-16,33,34)$. These humanrhesus chimeras have been proposed as anti-HIV-1 gene therapy candidates. Since potency is an essential consideration for any anti-HIV-1 therapy, hT5Cyp was compared side-by-side with the most promising human-rhesus chimeras. hTRIM $5 \alpha_{\mathrm{R} 332 \mathrm{P}}(16$, $33)$ and hTRIM5 $\alpha_{\text {rh323-332 }}(14,15)$ transgenes, stably expressed in CRFK cells, inhibited single-cycle HIV-1 vectors by about 10 -fold, approaching the anti-HIV-1 activity of rhTRIM5 $\alpha$ (Figure 5A and Supplemental Figure 2B). hT5Cyp, however, restricted HIV-1 almost 10 -fold more potently than even rhTRIM5 $\alpha$ (Figure 5A and Supplemental Figure 2B). In stable Jurkat $\mathrm{T}$ cell lines, the relative potency of hT5Cyp, and AoT5Cyp, was even more impressive (Figure 5B and Supplemental Figure 2A). Similar results were obtained in 293T cells stably expressing the indicated TRIM5 proteins (Supplemental Figure 2C).

bT5Cyp eliminates the spread of HIV-1 in tissue culture. Jurkat T cells stably transduced with TRIM5 expression vectors were infected with replication-competent HIV-1. hTRIM $5 \alpha_{\text {R332P }}$ and hTRIM $5 \alpha_{\text {rh } 323-332}$ had minimal effect on the kinetics of HIV-1 infection (Figure 5C). rhTRIM5 $\alpha$ delayed the HIV-1 replication peak by 8 days (Figure 5C). In contrast, HIV-1 replication was not detected in cells transduced with hT5Cyp or AoT5Cyp for the 2-month observation period (Figure 5, C and D). HIV-1 replication in cells transduced with hT5Cyp bearing a mutation that blocks binding to HIV-1 CA (CypA-H126Q; ref. 27) was as rapid as in cells transduced with empty vector (Figure 5, C and D). In the interest of identifying HIV-1 mutations that confer resistance to hT5Cyp, HIV-1 infection was initiated on 10 separate occasions, over a range of infectious doses, in coculture of hT5Cyp-expressing and untransduced cells, and by direct transfection of infectious HIV-1 proviral DNA into hT5Cyp-expressing Jurkat cells. No evidence of the spread of HIV-1 was detected in the presence of hT5Cyp.

New lentiviral vectors bearing $b$ T5Cyp block HIV-1 infection of primary cells. hT5Cyp-mediated restriction of HIV-1 in primary human CD4 ${ }^{+}$ $\mathrm{T}$ cells and macrophages was assessed next. To identify cells trans- 
A
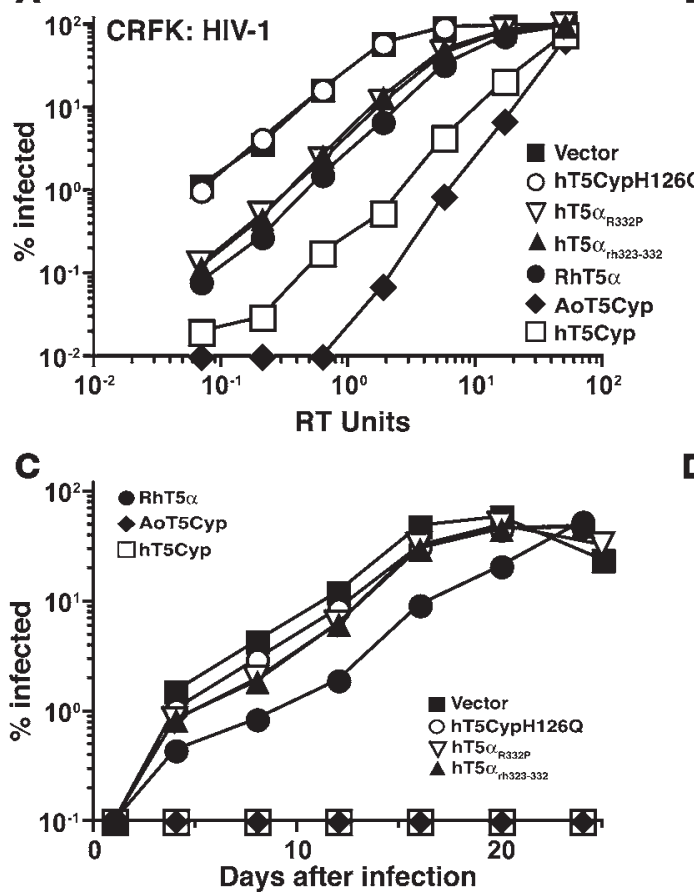

B

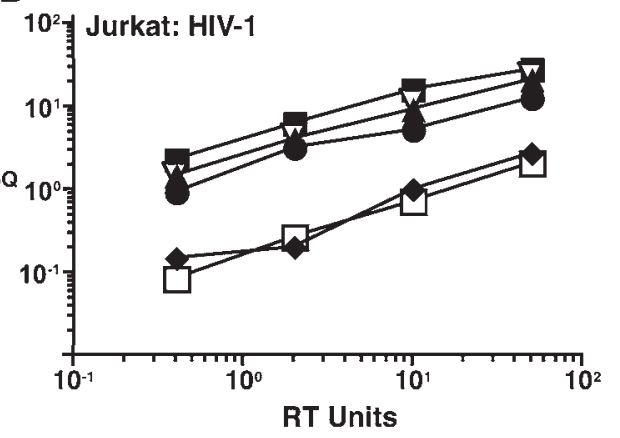

D

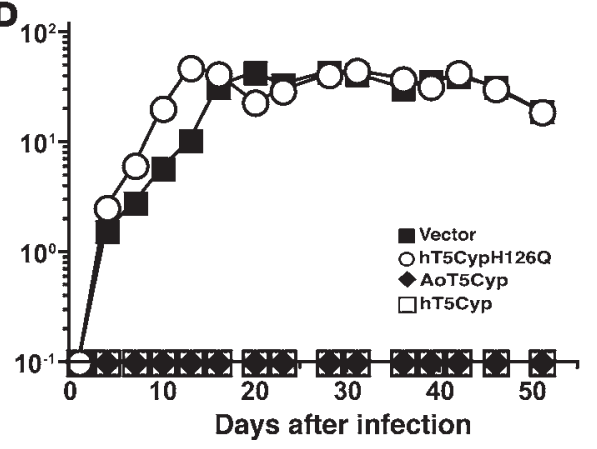

Figure 5

The anti-HIV-1 activity of hT5Cyp is superior to that of rhTRIM5 $\alpha$. (A and B) CRFK (A) and Jurkat $T$ cells $(B)$ were transduced with lentiviral vectors encoding the indicated TRIM5 proteins and a puromycin resistance cassette. Pools of puromycin-resistant cells were infected with increasing amounts of a single-cycle HIV-1-GFP vector. The percentage of $\mathrm{GFP}^{+}$cells was determined 48 hours later. (C and D) As in A and B, Jurkat $T$ cells were transduced with the indicated vectors and challenged with HIV-1 ${ }_{\text {NL4-3-GFP-IRES-Nef. The }}$ percentage of infected cells was determined by flow cytometry. duced with hT5Cyp, attempts were made to use a bicistronic lentiviral vector that directs synthesis of GFP from an internal ribosome entry site (IRES; Figure 6A). These vectors failed to generate GFP in primary $\mathrm{CD}^{+} \mathrm{T}$ cells (Figure $6 \mathrm{~B}$ ). Better results were obtained when a vector was engineered that bore 2 promoters, one from the spleen focus forming virus (SFFV) LTR and the other from the hCypA gene (Figure 6, A and B). Cosynthesis of 2 reporter genes, GFP and dsRED, within primary $\mathrm{CD}^{+} \mathrm{T}$ cells transduced with scALPS, was confirmed by flow cytometry (Supplemental Figure 3).

Primary $\mathrm{CD}^{+} \mathrm{T}$ cells transduced with dual-promoter vectors bearing hT5Cyp or the non-restrictive hT5CypH126Q were sorted based on GFP expression, and expression of the transgenes was confirmed by real-time RT-PCR (Supplemental Figure 3). Transduced cells proliferated at the same rate as untransduced cells, produced comparable amounts of IL-2 upon stimulation, and expressed the same levels of cell surface CD4, CXCR4, and MHC I (Supplemental Figure 4). Upon challenge with replication-competent, CXCR4-tropic HIV-1 $1_{\mathrm{NL} 4-3}$, viral replication in hT5CypH126Q-transduced cells peaked on day 12, and infection was not established in hT5Cyp-transduced cells (Figure 6C). The same hT5Cyp-mediated block to infection was observed with a primary isolate of HIV-1 (Figure 6D). Monocyte-derived macrophages were also transduced with vectors encoding hT5Cyp or hT5CypH126Q and sorted based on GFP expression. Cells were infected with $\mathrm{HIV}-1_{\mathrm{NL} 4-3}$, modified to be CCR5 tropic, at a high MOI $(\mathrm{MOI}=2)$. Upon decay of the input virus, macrophages transduced with hT5Cyp were clearly resistant to HIV-1 infection (Figure 6E). Furthermore, macrophages transduced with hT5Cyp-encoding vectors were resistant to a primary isolate of HIV-1 (Figure 6F).

bT5Cyp confers selective advantage to $\mathrm{CD} 4^{+} \mathrm{T}$ cells challenged with $H I V-1$. The previous experiments showed that HIV-1 infection was blocked when all cells in the culture had been transduced with the hT5Cyp vector. Next we evaluated anti-HIV-1 efficacy under suboptimal conditions, in which less than $25 \%$ of cells were transduced with hT5Cyp. In the absence of HIV-1 infection, the percentage of transduced cells persisting in the culture over the course of a month was equivalent for hT5Cyp and hT5CypH126Q. With HIV-1 infection, the hT5CypH126Q cells declined to less than $10 \%$ of the cells in the culture (Figure 6G). In contrast, hT5Cyp cells had a distinct advantage, expanding to $75 \%$ of the cells in the population (Figure 6G). This selective advantage was mirrored in prolonged cell viability within the cultures transduced with hT5Cyp-coding vector compared with those transduced with hT5CypH126Qexpressing vector (Supplemental Figure 5). The survival advantage was most likely due to the antiviral activity of hT5Cyp, since HIV-1 replication was reduced in cultures containing hT5Cyp cells (Figure 6G). Thus, our findings suggest that even when a small number of cells are transduced in culture conditions in which HIV-1 can spread directly from cell to cell, hT5Cyp is potent enough to block HIV-1 infection.

bT5Cyp protects against HIV-1 infection in vivo. To test the ability of hT5Cyp to protect against HIV-1 infection in vivo, 2 approaches analogous to those used in human gene therapy were taken. In one experimental model, human $\mathrm{CD}^{+} \mathrm{T}$ cells were adoptively transferred into Rag $2^{-1-} \mathrm{\gamma c}^{-/-}$mice. This mouse strain lacks B, T, and NK cells and does not reject xenografts (35). $\mathrm{CD}^{+} \mathrm{T}$ cells were transduced with the hT5Cyp or hT5CypH126Q vectors and sorted for GFP expression, leading to greater than $93 \%$ purity of $\mathrm{GFP}^{+}$input cells (Figure 7A). Age-matched 6- to 10-week-old Rag2 $2^{-1-} \mathrm{\gamma c}^{-1-}$ mice were conditioned with clodronate and sublethal irradiation (36) and injected intraperitoneally with $2.5 \times 10^{6}$ transduced CD4+ $\mathrm{T}$ cells (Figure 7B). Two and 6 weeks after transplant, peripheral blood engraftment was assessed by measuring the percentages of human $\mathrm{CD} 5^{+} \mathrm{CD}^{+} \mathrm{GFP}^{+}$cells. We found that $50 \%$ to $90 \%$ were $\mathrm{GFP}^{+}$at both time points (2-week mean \pm SEM: hT5Cyp, $81.9 \% \pm 4.5 \%$, $n=8$; hT5CypH126Q, 73.4\% \pm 4.4\%, $n=8$; 6-week mean \pm SEM: 
A

AIG:

\begin{tabular}{|c|c|c|c|c|}
\hline \multicolumn{3}{|l|}{$\overrightarrow{S F F V}>\widehat{h T S}$} & IRES & \\
\hline
\end{tabular}

SCALPS:

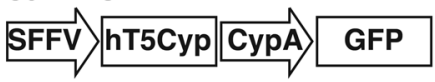

B
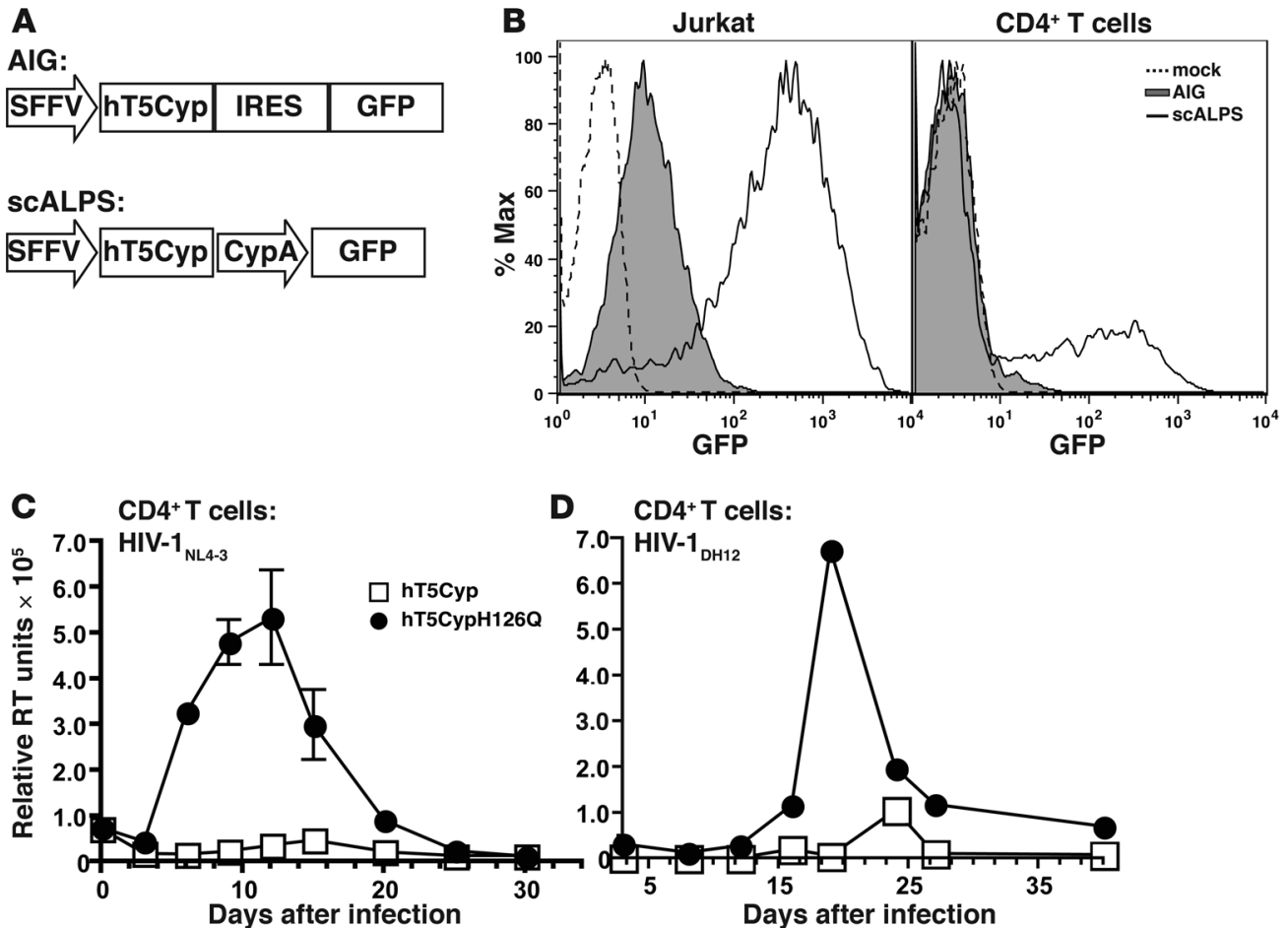

D CD4 ${ }^{+} T$ cells:

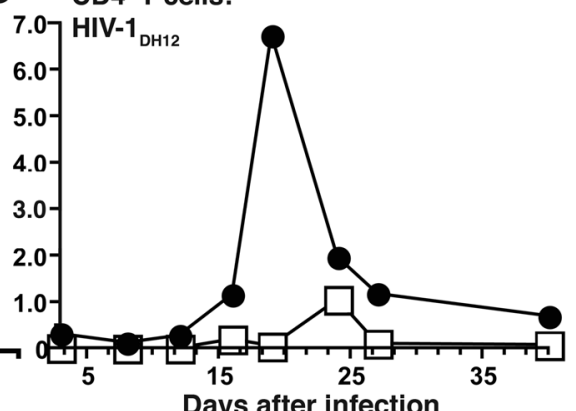

E Macrophages:

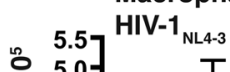

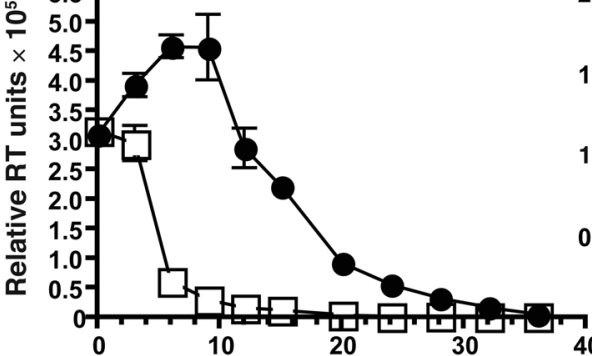

Days after infection

G

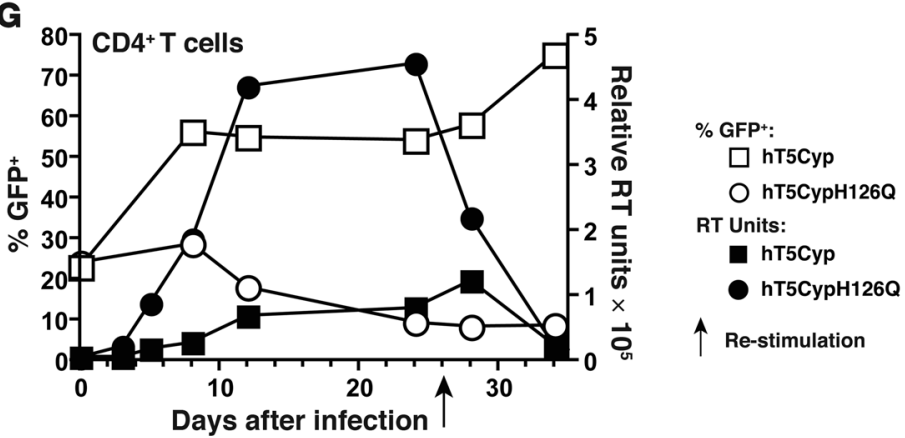

Figure 6

New lentiviral vectors bearing hT5Cyp potently block replication-competent HIV-1 in primary cells. (A) Design of conventional bicistronic (AIG) and new dualpromoter (SCALPS) lentiviral vectors. (B) Forty-eight hours after transduction with AIG and scALPS vectors, Jurkat T cells and primary $\mathrm{hCD} 4{ }^{+} \mathrm{T}$ cells were analyzed by flow cytometry for GFP. (C and D) Primary human $\mathrm{CD} 4{ }^{+} \mathrm{T}$ cells transduced with scALPS encoding the indicated hT5Cyp proteins were sorted for GFP and infected with lab clone HIV-1 ${ }_{\mathrm{NL} 4-3}(\mathbf{C})$ or primary isolate HIV-1 $1_{\mathrm{DH} 12}$ (D), and RT activity in the supernatant was measured. Triplicate spreading infection for 1 representative donor of 4 is shown. (E and $\mathbf{F}$ ) Human macrophages generated from GM-CSF-treated, CD14+ monocytes were transduced with the indicated vectors, sorted for GFP, and challenged with modified HIV-1 $1_{\text {NL4-3 }}$ bearing a CCR5-tropic Env (E) or primary isolate HIV-1 $1_{132 \mathrm{~W}}$ (F). Infection was monitored as described in $\mathbf{C}$ and $\mathbf{D}$. Error bars in $\mathbf{C}$ and $\mathbf{E}$ represent SEM. $n=3$. (G) hT5Cyp-bearing $\mathrm{CD}^{+}{ }^{+} \mathrm{T}$ cells exhibit a selective advantage after challenge with HIV-1. CD4+ $T$ cells were transduced with SCALPS encoding the indicated T5Cyp proteins. Cultures containing $23 \%$ GFP+ cells were challenged with HIV-1 NL4-3 $_{\text {S }}$ (15 ng p24/106 cells), and the percentage of GFP+ cells was monitored (left $y$ axis, open symbols). Supernatant RT activity was measured (right $y$ axis, filled symbols). Cultures were restimulated using allogeneic PBMCs, IL-2, and PHA 26 days following infection (arrow).
hT5Cyp 76.1\% $\pm 6.0 \%, n=8$; hT5СypH126Q, 76.8\% $\pm 1.56 \%, n=8$ ), indicating that GFP percentages remained stable and engraftment efficiency was not altered by hT5Cyp or hT5CypH126Q. Similar to peripheral blood, percentages of engraftment and $\mathrm{GFP}^{+}$cells did not differ in lymphoid organs between the 2 constructs used (Figure 7C). However, we consistently observed that GFP expression was higher in peripheral blood, bone marrow, and spleen compared with somewhat lower levels of GFP expression in thymus and mesenteric lymph nodes (Supplemental Figure 6). The reasons for this are unclear. One can speculate that (a) the minor fraction of T cells that were GFP low or GFP negative, the latter not transduced at transfer $(<7 \%$, Figure $7 \mathrm{~A})$, had different biologic properties (e.g., better homing capacity to lymphoid organs as thymus and lymph nodes) compared with $\mathrm{GFP}^{+}$cells; (b) GFP expression correlates with activation status of transduced initially-GFP ${ }^{+}$ cells, with metabolically less active cells expressing lower levels or lacking GFP expression in thymus and mesenteric lymph nodes, an issue that needs to be clarified in further studies. 
A Day 0:

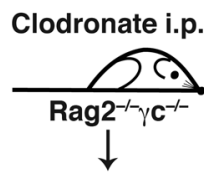

Day 1:

4 Gy; $2.5 \times 10^{6}$ transduced $\mathrm{CD}^{+} \mathrm{T}$ cells i.p.

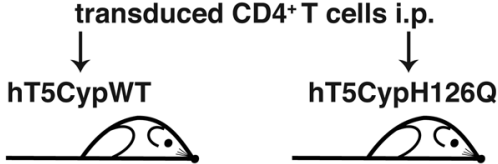

Week 6:

Analysis

B

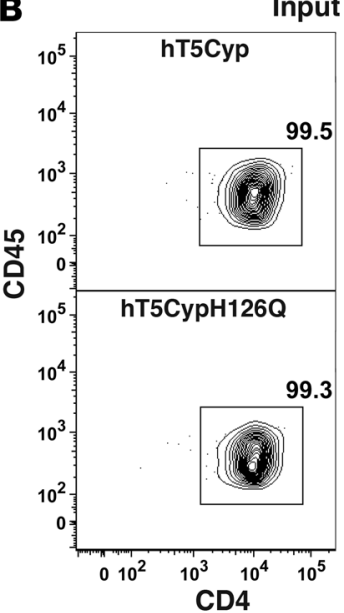

nput CD4+ cells

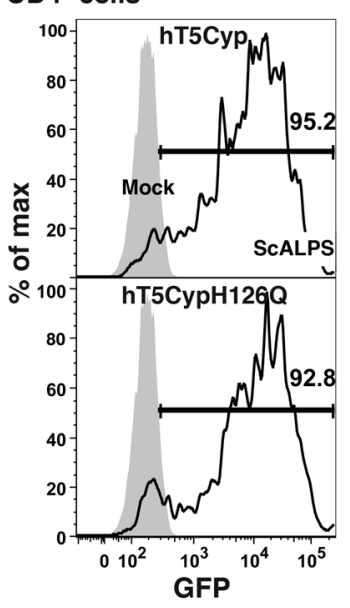

C

Thymus 6 wk after transplant
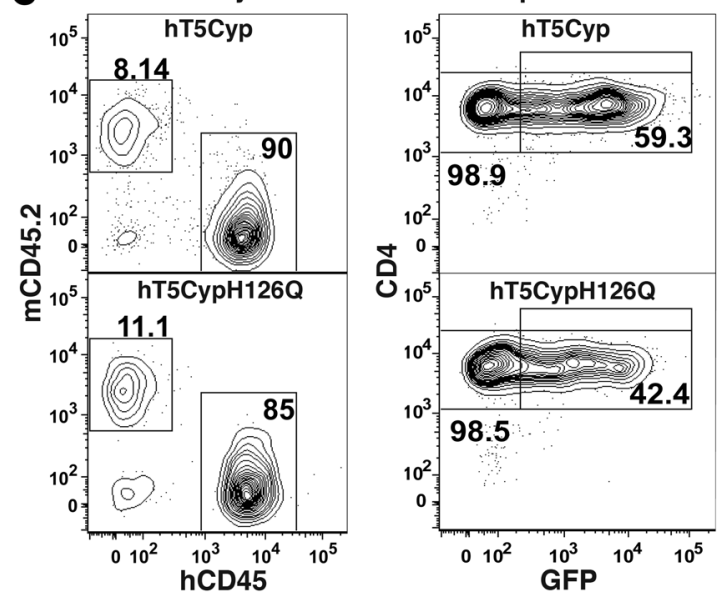

To assess the effect of hT5Cyp in vivo, mice were infected with HIV-1 after adoptive transfer of transduced $\mathrm{CD} 4^{+} \mathrm{T}$ cells. When mice received hT5Cyp-transduced $\mathrm{CD}^{+}{ }^{+} \mathrm{T}$ cells, 2 weeks after HIV-1 infection the $\mathrm{CD}^{+}$cells constituted $6 \%( \pm 2.87 \%)$ of mean nucleated peripheral blood cells (Figure $8 \mathrm{~A})$. This was 34-fold higher than the percentage of $\mathrm{CD}^{+}$cells observed in mice transplanted with hT5CypH126Q-transduced CD4 ${ }^{+} \mathrm{T}$ cells (mean, $0.18 \% \pm 0.04 \%$ ), a difference that was statistically significant $(P=0.0002, n=10$ hT5Cyp, $n=8$ hT5CypH126Q) (Figure 8A). A 9-fold reduction in mean plasma viral load was observed when mice engrafted with hT5Cypexpressing $\mathrm{CD}^{+}$cells were compared with mice engrafted with hT5CypH126Q-expressing CD4 ${ }^{+}$T cells $(P=0.005 ; n=8$ hT5Cyp,

\section{Figure 7}

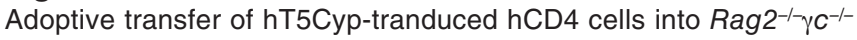
mice. Human peripheral blood CD4+ $\mathrm{T}$ cells transduced with lentiviral vector scALPS bearing either hT5Cyp or hT5CypH126Q were sorted for GFP and injected into 6- to 10-week-old $\mathrm{Rag}^{-1-} \mathrm{\gamma C}^{-/-}$mice. The experimental design is shown schematically in A. (B) Flow cytometric analysis of the transduced, sorted cells prior to adoptive transfer. Left: Nearly all cells were hCD4+. Right: GFP fluorescence in transduced cells (open histograms, scALPS) or in untransduced, donor-identical CD4+ T cells (grey histogram; mock). (C) Six weeks after transplant, single-cell suspensions of thymus were analyzed for human cell engraftment and transgene expression. Left: Recipient-derived CD45+ mouse cells (mCD45.2) and donor-derived hCD45+ cells. Right: CD4 and GFP signals for the hCD $45^{+}$cells. In $\mathbf{B}$ and $\mathbf{C}$, the numbers indicate the percentage of cells within the gates.

$n=8$ hT5CypH126Q) (Figure 8B). These results indicate that hT5Cyp inhibits HIV-1 replication and protects against HIV-1-associated destruction of $\mathrm{CD}^{+} \mathrm{T}$ cells in vivo.

Further evidence for the in vivo effects of hT5Cyp was sought by examining lymphoid organs of mice infected with HIV-1. Three weeks after transplant, single-cell suspensions were analyzed for the human T cell marker CD3, the hT5Cyp vector reporter GFP, and HIV-1 p24 antigen. Few if any hT5Cyp-transduced human $\mathrm{T}$ cells isolated from thymus or lymph node of HIV-1-infected mice were $\mathrm{p}^{2} 4^{+}$, as compared with $1.5 \%-6.5 \%$ of the cells transduced with hT5CypH126Q (Figure 8C). Consistent with fewer productively infected $\mathrm{T}$ cells, downregulation of cell surface CD4 was not observed with hT5Cyp-bearing cells (37) in either the thymus or the mesenteric lymph node of mice infected with CXCR4tropic or CCR5-tropic HIV-1 (Figure 8D).

Tissue sections of thymus and mesenteric lymph node were directly examined for human $\mathrm{CD}^{+} \mathrm{T}$ cells and HIV-1 p24 antigen. The engraftment of the CD4 ${ }^{+} \mathrm{T}$ cells transduced with hT5Cyp and hT5CypH126Q was similar (Figure 8E). Following HIV-1 infection, intense p24 staining was observed in thymic tissue sections from animals engrafted with hT5CypH126Q-transduced $\mathrm{CD}^{+} \mathrm{T}$ cells but not in tissues from mice engrafted with $\mathrm{CD}^{+}$ $\mathrm{T}$ cells transduced with hT5Cyp (Figure 8E). Similar results were obtained for tissue sections from mesenteric lymph nodes (Supplemental Figure 7). These experiments show that hT5Cyp significantly reduced or prevented productive HIV-1 infection in vivo. Given these findings, we speculate that most of the human T cells in the thymus and lymph node were indeed transduced, although levels of GFP expression were lower compared with blood, bone marrow, and spleen $\mathrm{CD}^{+}$cells.

In the second in vivo approach to study the effects of hT5Cyp on HIV-1 replication, CD $34^{+}$hematopoietic progenitor cells transduced with vectors bearing hT5Cyp or hT5CypH126Q were transplanted into preconditioned neonatal Rag2 $2^{-/-} \mathrm{\gamma C}^{-/}$mice (38), leading to $\mathrm{B}$ and $\mathrm{T}$ cell reconstitution in peripheral blood 8 weeks after transplant. The percentage of $\mathrm{GFP}^{+}$cells among the $\mathrm{CD} 45^{+}$ lymphocytes and CD $19^{+} \mathrm{B}$ cells that developed in the mice was similar to the percentage of $\mathrm{GFP}^{+}$cells among the input $\mathrm{CD} 34^{+}$ cells, but development of $\mathrm{GFP}^{+} \mathrm{CD}^{+} \mathrm{T}$ cells was rarely observed (Supplemental Figure 8A). Nonetheless, mice with hCD $45^{+}$ cells in peripheral blood were challenged with HIV-1. At 25 days following infection, mean viremia in mice reconstituted with $\mathrm{CD} 4^{+}$cells bearing hT5Cyp was less than $30 \%$ the mean viremia of the hT5CypH126Q control mice (Supplemental Figure 8B; 
A

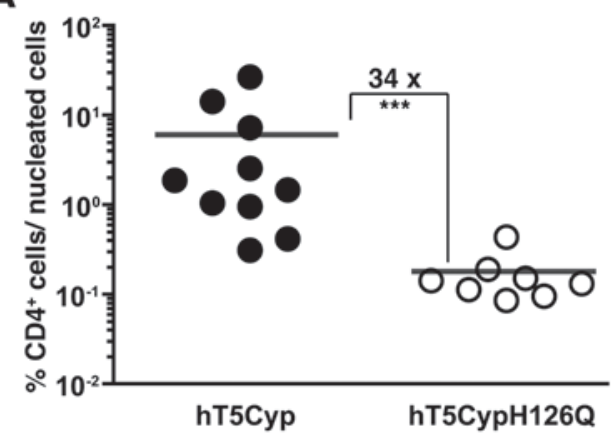

C

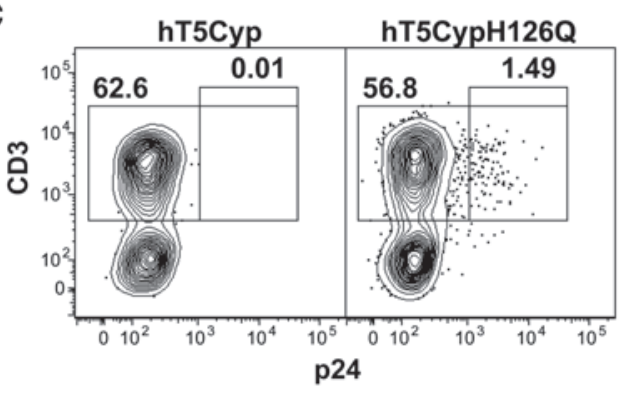

B

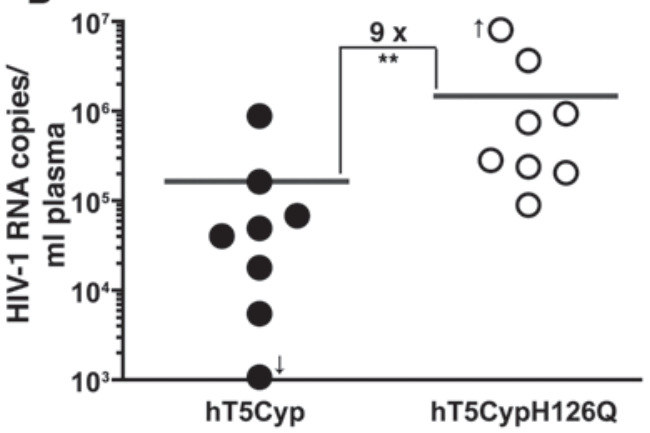

D

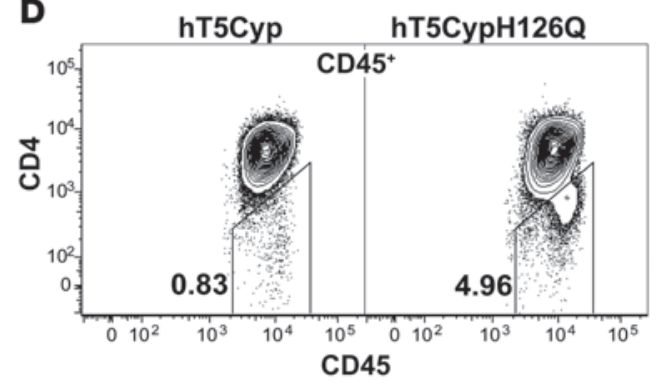

$\alpha$ p24

ap24

E

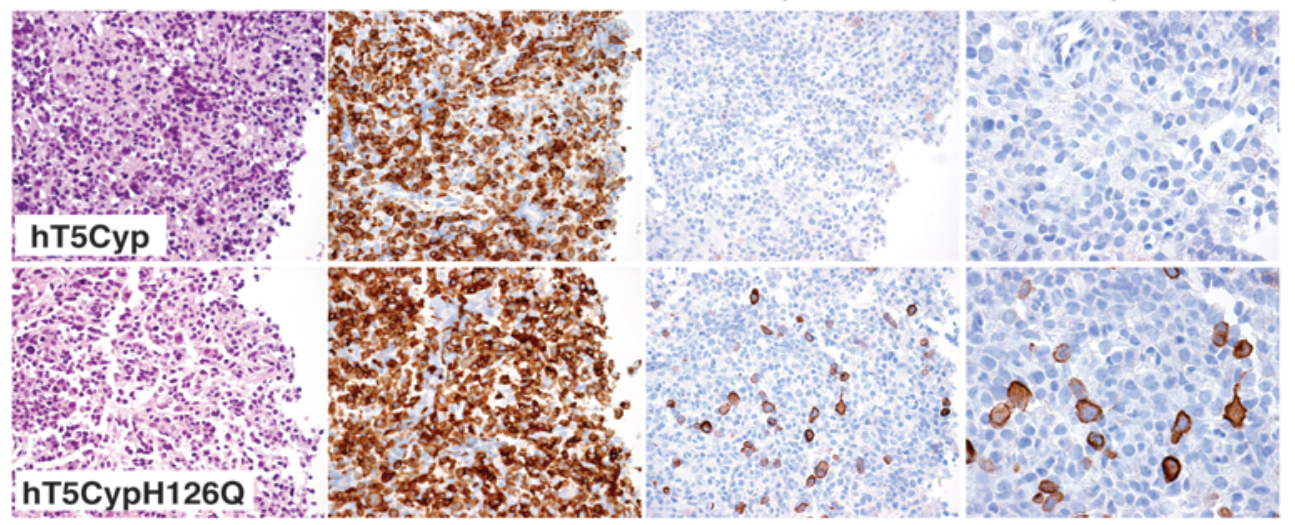

\section{Figure 8}

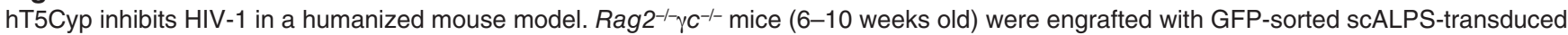
CD4+ ${ }^{+}$cells expressing either hT5Cyp or hT5CypH126Q. (A) Five days after engraftment, mice were challenged with HIV-1 ${ }_{\mathrm{NL} 4-3}$. The percentage of hCD4+ $\mathrm{T}$ cells among all nucleated cells in the peripheral blood of individual mice is shown at 2 weeks following infection. The fold difference for the mean is indicated (horizontal bars, ${ }^{* \star} P=0.0002$, Mann-Whitney $U$ test). (B-E) Two weeks after engraftment, mice were infected

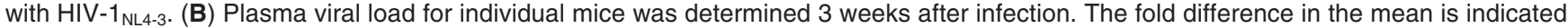
(horizontal bars; ${ }^{\star *} P=0.005$, Mann-Whitney $U$ test). (C and D) Three weeks after infection, single-cell suspensions from thymus were analyzed by flow cytometry for p24 and hCD3 cells (C) or hCD45 and hCD4 (D). The numbers indicate the percentage of cells within the gates. (E) Paraffin-embedded sections of thymus are shown, stained with H\&E, anti-hCD3, or anti-p24, as indicated. All panels are at $\times 40$ magnification, except the 2 right panels, which are $\times 80$ magnification. In $\mathbf{C}-\mathbf{E}$, representative results from 3 sets of experiments are shown.

$n=4$ hT5Cyp, $n=3$ hT5CypH126Q). Moreover, while peripheral blood engraftment of hCD $45^{+}$remained unchanged or declined over the 25 days of HIV-1 infection, $\mathrm{GFP}^{+} \mathrm{CD} 45^{+}$cells selectively expanded in the context of hT5Cyp but not of hT5CypH126Q (Supplemental Figure 8C).

\section{Discussion}

Anti-HIV-1 gene therapies have generally modified host cell factors required for viral replication or inhibited essential viral elements (4). Here, a third approach was adopted: the exploitation of natural inhibitors that evolved over millions of years in primates in response to retroviral attack $(14-16,33)$. The potent block to HIV-1 observed with AoT5Cyp inspired the design of a human equivalent, hT5Cyp, that robustly blocks HIV-1 in vitro and in vivo. A distinct T5Cyp fusion gene was generated by an independent retrotransposition event in Old World macaques. In this case, restriction activity was detected against HIV-2 and FIV, but not against HIV-1 (39-43). The convergent evolution of T5Cyp fusion proteins with distinct retroviral specificities indicates a strong selection for these potent restriction factors. While the specific force behind selection 
remains to be elucidated for individual TRIM5 orthologs, in each case the selective pressure is likely to have been a retrovirus (15).

Because TRIM5 $\alpha$ and T5Cyp are modular proteins, it was expected that the engineering of a restrictive hT5Cyp would not be difficult. Surprisingly, only 3 of 13 hT5Cyp fusions inhibited HIV-1 with results comparable to AoT5Cyp. No correlation was observed between protein levels and antiviral activity, and CA-binding activity was not sufficient to restrict HIV-1 (Figure 2, A and B). AntiHIV-1 activity was observed when hCypA was fused at the apex of the hTRIM5 $\alpha$ PRYSPRY domain in the structural model (Figure 2C). Analysis of nonsynonymous mutations indicates that this region undergoes some of the strongest selective pressure in the primate lineage, and functional experiments pinpoint it as a specificity determinant for CA recognition $(15,16,44)$. CypA fusion to this site may have antiviral activity because this apical loop is uniquely situated for coordinating CA recognition and effector domains of TRIM5. Correlation between restriction activity and the ability of hT5Cyp fusion proteins to form cytoplasmic bodies suggests that activity requires particular spatial constraints or association with unknown cofactors. The strict structural requirements for engineering a functional hT5Cyp emphasize the remarkable nature of the fusion gene that was generated by retrotransposition in New World owl monkeys (7).

Optimal gene therapy candidates provide high efficacy and low antigenicity. Hematopoietic progenitors transduced with rhesus macaque TRIM $5 \alpha$ or human-rhesus TRIM5 $\alpha$ chimeras differentiate into HIV-1-resistant macrophages and T cells $(34,45)$. These proteins, however, are potentially antigenic, which could lead to the elimination of modified cells in vivo (22). Nonetheless, efforts have been taken to minimize potential sources of antigenicity. By substituting only critical amino acids within the hTRIM5 $\alpha$ PRYSPRY domain (hTRIM $5 \alpha_{\mathrm{R} 332 \mathrm{P}}$, hTRIM $5 \alpha_{\mathrm{rh} 332-332}$ ) one can reduce the risk of antigenicity, but this also decreases anti-HIV-1 efficacy (Figure 5). hT5Cyp is the most potent HIV-1 inhibitor of the TRIM5 proteins we compared, including rhTRIM5 $\alpha$, in both single-cycle and spreading infections of human T cells with HIV-1 (Figure 5). This difference in activity might be due to the apparently higher CA affinity of the Cyp domain of hT5Cyp (46). In addition to its superior potency, hT5Cyp is composed of human components with no amino acids derived from non-human orthologs. Despite having what we believe is a novel fusion junction, hT5Cyp is likely to have the lowest antigenic potential among these TRIM5-based gene therapies.

HIV-1 rev, tat, and gag $(4,47,51)$, as well as the HIV-1 co-receptor CCR5 (48-50), have been targeted by various means including siRNAs $(4,50,51)$ and zinc finger endonucleases (49). With either approach there are concerns regarding toxicity (51), off-target effects (48), and viral resistance $(4,51)$. Transdominant revM10 proteins have been tested in patients without reducing viremia (47). Furthermore, revM10-resistant HIV-1 strains were observed in vitro (52). Similarly, for siRNA approaches, escape mutants arise readily, since single-nucleotide changes are sufficient to escape the siRNA-mediated blocks to HIV-1 replication (51). CCR5 disruption, one of the most promising approaches to date, could select for CCR5-independent viruses (53) and is not without consequence, as the CCR5 $\Delta 32$ allele is a risk factor for fatal West Nile Virus infection (54).

hT5Cyp is a broadly acting, antilentiviral agent that blocks CCR5- and CXCR4-tropic HIV-1 clones and primary isolates as well as some HIV-2, SIV, and FIV clones (Figures 4 and 6). TRIM5 isoforms form hetero-multimers, and hT5Cyp might interfere with endogenous hTRIM5 $\alpha$ function (55). However, no significant decrease in hTRIM $5 \alpha$-mediated restriction of N-MLV, and no alteration in cell surface markers or cytokine secretion, was observed in hT5Cyp-transduced cells (Supplemental Figure 4). Despite extensive efforts to identify hT5Cyp-resistant viruses, none were detected, perhaps due to the potency of the antiviral activity and the fact that hT5Cyp blocks HIV-1 prior to RT, thus precluding the generation of the genetic diversity required for emergence of resistance. hT5Cyp additionally blocked a natural HIV-1 CA variant reported to be resistant to AoT5Cyp (28) (Figure $3 \mathrm{D})$. These results suggest that, if hT5Cyp were utilized as antiHIV-1 gene therapy in people, viral resistance would not emerge readily and host cell function would remain intact.

The evaluation of HIV-1 therapies is limited by a lack of adequate animal models for HIV-1 replication and pathogenesis. The hCD4 $4^{+}-\mathrm{Rag}^{-1-} \mathrm{\gamma c}^{-/-}$mouse developed here is a straightforward alternative to the HIV-1 mouse models used to date (56-59). It permitted high-level HIV-1 viremia and assessment of the effect of hT5Cyp transduction on HIV-1 infection and CD4 ${ }^{+} \mathrm{T}$ cell protection (Figure 8). Autotransfusion of ex vivo expanded CD4 ${ }^{+} \mathrm{T}$ cells is of clinical benefit to HIV-1-infected people (60). Human CD4 ${ }^{+}$ $T$ cells can be transduced at high efficiency and expanded to more than $10^{9}$ cells ex vivo. Given that hT5Cyp-transduced CD4 $4^{+}$cells can be expanded in vitro and in vivo (Figures 6 and 7), and that these cells exhibit a selective advantage during HIV-1 infection (Figure 6G and Figure 8A), there might be an impressive effect of hT5Cyp transduction on autologous $\mathrm{CD}^{+} \mathrm{T}$ cell gene therapy in the clinical setting.

$\mathrm{CD} 34^{+}$hematopoietic stem cells transduced with the state-ofthe-art lentiviral vectors described here and elsewhere (61) achieve long-term engraftment of Rag2 $2^{-/} \gamma^{-/-}$mice, but transgene expression in the mature $\mathrm{CD}^{+} \mathrm{T}$ cells that develop within these animals has not been consistently detected. Nonetheless, hT5Cyp modestly reduced HIV-1 viremia in humanized $\mathrm{Rag}^{-/-} \mathrm{CC}^{-/-}$mice (Supplemental Figure 8). With improved transduction methods, it will be important to examine the effect of stem cell transduction with hT5Cyp on immune cell development and HIV-1 infection within one of the humanized mouse models currently under development aimed at enhancing de novo $T$ cell development and in vivo immune function $(62,63)$.

\section{Methods}

Plasmids and cloning. Most HIV-1 plasmids and vectors have been described previously (64). pNL4-3 contains a complete infectious HIV-1 provirus. pNL4-3-R5, a gift from F. Kirchhoff (University of Ulm, Ulm, Germany), bears the V3 loop of the CCR5-tropic 92TH014-2 HIV-1 strain (65). To generate pNL4-3-GFP-IRES-Nef, EGFP and the encephalomyocarditis virus (ECMV) IRES were inserted upstream of nef. pNLAenvGFP is pNL4-3 with an $e n v$-inactivating mutation and EGFP in place of $n e f$. CSGW is an HIV-1 vector expressing EGFP under the control of the SFFV promoter. pFUPI, engineered from pFUW (66), uses the ubiquitin promoter to drive expression of a puromycin resistance cassette, followed by the ECMV IRES (Supplemental Figure 1A).

hT5Cyp variants were cloned by overlapping PCR using the oligonucleotides shown in Supplemental Table 1. rhTRIM5 $\alpha$, hTRIM5 $\alpha_{\text {R332P }}(16$, $33)$ and hTRIM5 $\alpha_{\text {rh323-332 }}(14,15)$ were gifts from J. Sodroski and F. DiazGriffero (Harvard University, Boston, Massachusetts, USA). All TRIM5 constructs were cloned downstream of the IRES in FUPI. All PCR products were sequenced to eliminate unwanted mutations. 
pAIG uses the SFFV promoter to drive expression of TRIM5, followed by IRES EGFP. In pscALPS, the SFFV promoter drives expression of TRIM5 and the human CypA promoter drives expression of EGFP. For 3-component vector production, HIV-1 gag and pol were expressed using psPAX2, p8.9N $\Delta$ SB, p8.9N $\Delta$ SB-G89V or p8.9N $\Delta$ SB-V86P/H87Q/I91V/M96I. The V86P/H87Q/I91V/M96I mutation in CA (28) was provided by P. Gallay (The Scripps Research Institute, La Jolla, California, USA). $\operatorname{SIV}_{\mathrm{mac}} 251$ vectors were produced using GAE1.0 and the packaging vector SIV3 ${ }^{+}$(67). SIV $_{\text {AGM }}$ tan and HIV-2 $2_{\text {ROD }}$ vectors each have an env-deletion and EGFP in place of nef(9). FIV vectors were produced using the genomic vector pGINSIN and the packaging vector pFP93 (30). All lentiviral vectors were pseudotyped using the vesicular stomatitis virus glycoprotein (VSV-G). For biochemistry, hT5Cyps were cloned into the p3xFLAG-CMV-7.1 expression vector (Sigma-Aldrich), and HIV-1 CA was expressed as a GST fusion using the $\mathrm{EF} 1 \alpha$ promoter.

Cells. Human embryonic kidney cells (293T) and CRFK feline kidney cells were maintained in DMEM supplemented with 10\% FBS and glutamax (Invitrogen). Jurkat E6-1, H9, CEM-SS, and SUP-T1 T cell leukemia/lymphoma cell lines and U937 promonocytic lymphoma, and THP-1 monocytic leukemia cells were grown in RPMI-1640 with 10\% FBS and glutamax (Invitrogen). For creation of TRIM5-expressing cell lines, suspension cells were transduced with $2 \times 10^{5} \mathrm{Jurkat}$ infectious units $/ \mathrm{ml}$ and adherent cells were transduced with $2 \times 10^{4}$ Jurkat infectious units $/ \mathrm{ml}$ of FUPI containing TRIM5. Jurkat infectious units were determined by serial dilutions of concomitantly produced FUPI-GFP on Jurkat E6 cells. At 48 hours after transduction, cells were selected with puromycin. The different constructs were normalized to each other by comparing RT activity of the supernatants and assessing the puromycin-resistant colonies after serial dilution on HeLa cells. CD $4^{+} \mathrm{T}$ cells were enriched from PBMCs (>99\% CD4 $\left.4^{+}\right)$using positive selection magnetic beads (Miltenyi Biotec) and stimulated for 24 hours on multisorb 96-well plates (Nunc) coated with $2 \mu \mathrm{g} / \mathrm{ml}$ antiCD3 and $2 \mu \mathrm{g} / \mathrm{ml}$ anti-CD28 antibodies (BD Biosciences) in RPMI with $10 \% \mathrm{FBS}$, glutamax (Invitrogen), and $20 \mathrm{IU} / \mathrm{ml} \mathrm{hIL-2}$. Where indicated in the figure legends, and in preparation for adoptive transfer to mice, $\mathrm{CD} 4^{+}$ $\mathrm{T}$ cells were stimulated using allogeneic PBMCs irradiated at $50 \mathrm{~Gy}$ in the presence of $20 \mathrm{IU} / \mathrm{ml} \mathrm{IL-2}$ and phytohemagglutinin (PHA) at $1 \mu \mathrm{g} / \mathrm{ml}$. Cells were washed and replated 24 hours prior to viral transduction. CD $14^{+}$ monocytes were enriched from PBMCs ( $\left.>99 \% \mathrm{CD} 14^{+}\right)$using positive-selection magnetic beads (Miltenyi Biotec). CD14+ monocytes were resuspended in complete RPMI supplemented with $50 \mathrm{ng} / \mathrm{ml} \mathrm{recombinant} \mathrm{human} \mathrm{GM-}$ CSF (Leukomax; Novartis) at $1 \times 10^{6}$ cells $/ \mathrm{ml}$. CD $14^{+}$monocytes $\left(2 \times 10^{6}\right)$ were plated in each well of a 6 -well plate (Nunc) and allowed to differentiate into macrophages for 10 days before transduction. Transduction of human macrophages was performed as follows: cells were pretreated for 3 hours with SIV virus-like particles (50\% per volume, made with a 7:1 ratio of SIV3 ${ }^{+}$vector/pMD2.G) prior to viral transduction. For both $\mathrm{CD}^{+} \mathrm{T}$ cells and monocyte-derived macrophages, cells were sorted for GFP expression by FACS 72 hours after transduction.

Drugs. Unless otherwise noted, all chemicals were obtained from SigmaAldrich. Puromycin was used at $1 \mu \mathrm{M}$. CsA (Bedford Laboratories) was diluted to $10 \mathrm{mM}$ in DMSO and then further to $2.5 \mu \mathrm{M}$ in tissue culture media. $\mathrm{As}_{2} \mathrm{O}_{3}$ was made at $100 \mathrm{mM}$ in $1 \mathrm{~N} \mathrm{NaOH}$ and then further diluted to $1 \mathrm{mM}$ in PBS. The $\mathrm{pH}$ was adjusted to 7.5 with hydrochloric acid and stored at $4^{\circ} \mathrm{C}$.

Virus production. Vectors and viruses were produced by transfection of 293T cells using Lipofectamine 2000 (Invitrogen). Two-part vectors were produced by cotransfection of viral genome and VSV-G plasmids at a ratio of 7:1. Three-part vectors were produced by cotransfection of viral genome, gag-pol, and VSV-G plasmids at a ratio of 4:3:1. Infectious viruses were produced by transfection of $90 \%$ confluent $293 \mathrm{~T}$ cells in a T75 flask
(Nunc) with $40 \mu \mathrm{g}$ of pNL4-3, pNL4-3-R5, or pNL4-3-GFP-IRES-Nef. Supernatants containing viruses and vectors were cleared by centrifugation at $400 \mathrm{~g}$, filtered $(0.45 \mu \mathrm{m}$; Pall Acrodisc), tested for exogenous RT activity (7), titered on Jurkat E6 cells in single-cycle assay (7), and stored at $-80^{\circ} \mathrm{C}$. When indicated in the figure legends, the content of viral p24 antigen was quantified by an HIV-1 p24 ELISA kit (NIH AIDS Research and Reference Reagent Program).

Infections. Adherent cells $\left(2 \times 10^{4} /\right.$ well $)$ or suspension cells $\left(8 \times 10^{4} /\right.$ well $)$ were plated in 48-well plates (Nunc) for challenge with single-cycle GFP reporter vectors that were titered as indicated. GFP synthesis was assessed by flow cytometry 48 hours after infection. For spreading infection, $10^{6} \mathrm{Jurkat}$ cells were infected with $1-15 \mathrm{ng}$ p $24 / 10^{6}$ cells of HIV- $1_{\text {NL4-3-GFP-IRES-Nef. }}$ Cells were split every 3 days, fixed in $0.5 \%$ paraformaldehyde/PBS, and assayed for GFP synthesis by flow cytometry. Primary CD $4^{+} \mathrm{T}$ cells $\left(10^{6}\right)$ or macrophages were infected with $15 \mathrm{ng}$ p $24 / 10^{6}$ cells of CXCR4- or CCR5-tropic HIV- $1_{\text {NL4-3 }}$ stocks, or with low-passage primary isolates HIV-1 $1_{\mathrm{DH} 12}$ and HIV-1 $1_{132 \mathrm{~W}}$ (a gift from Olivier Schwartz, Pasteur Institute, Paris, France). Infection was monitored by assessing the accumulation of RT activity in the supernatant (7). FACSCalibur, Cellquest Pro (BD), and FlowJo software (Treestar Inc.) were used to record and analyze fluorescence. We acquired between $10^{5}$ and $5 \times 10^{5}$ events for analysis.

Western blotting and immunoprecipitation. To detect hT5Cyp, $5 \times 10^{6} 293 \mathrm{~T}$ cells were transfected with $20 \mu \mathrm{g}$ of each triple-FLAG-hT5Cyp plasmid using Lipofectamine 2000 (Invitrogen). Cells were lysed at 48 hours in Triton lysis buffer (50 mM Tris- $\mathrm{HCl} \mathrm{pH} 7.4,150 \mathrm{mM} \mathrm{NaCl}, 1 \%$ Triton X-100, with Complete Mini protease inhibitor cocktail [Roche]), cleared at $15,000 \mathrm{~g}$ for 5 minutes, incubated for 4 hours at $4^{\circ} \mathrm{C}$ with $2 \mu \mathrm{g} / \mathrm{ml}$ anti-FLAG M2 monoclonal antibody (Sigma-Aldrich), and then incubated 1 hour at $4^{\circ} \mathrm{C}$ with $20 \mu$ l of protein G-Sepharose beads (GE Healthcare). The beads were washed 3 times with $1 \mathrm{ml}$ Triton Lysis Buffer, and $20 \mu \mathrm{l}$ immunoprecipitate was subjected to SDS-PAGE on a $12 \%$ gel, transferred to a PVDF membrane, and immunoblotted with rabbit polyclonal antibody to CypA (Biomol).

For coimmunoprecipitation of hT5Cyp with HIV-1 CA, $5 \times 10^{6} 293 \mathrm{~T}$ cells were cotransfected as described above with $20 \mu \mathrm{g}$ p3xFLAG hT5Cyp and $20 \mu \mathrm{g}$ pEF1-GST-CA. At 48 hours, cells were lysed with RIPA buffer (50 mM Tris- $\mathrm{HCl}$ pH 8.0, $150 \mathrm{mM} \mathrm{NaCl}, 1 \%$ IGEPAL CA-630, 0.1\% SDS, $0.5 \%$ sodium deoxycholate, with Complete Mini protease inhibitor cocktail [Roche]) and accelerated at $15,000 \mathrm{~g}$ for 5 minutes. Lysate was divided into 2 equal parts and incubated on ice for 30 minutes with $20 \mu \mathrm{M} \mathrm{CsA} / \mathrm{DMSO}$ or an equivalent volume of DMSO for 30 minutes. Lysates were incubated with $30 \mu$ l glutathione-Sepharose beads (GE Healthcare) for 1 hour at $4^{\circ} \mathrm{C}$. Beads were washed 3 times with RIPA buffer. Proteins were subjected to SDS-PAGE and immunoblotted with rabbit polyclonal anti-CypA antibody (Biomol) and goat polyclonal anti-HIV-1 CA (NCI).

Structural model of the hTRIM5 $\alpha$ PRYSPRY domain. StruPro was used to superimpose and align the crystal structures for PRYSPRY-19q13.4.1 (Protein Data Bank [PDB] entry 2FBE; residue range, 11-184 of chain A), GUSTAVUS (PDB entry 2FNJ; residue range, $35-83$ and 88-233 of chain A), and TRIM21 (PDB entry 2IWG; residue range, 2-182 of chain $\mathrm{B})$, using an $\alpha$ carbon cutoff distance of $3.5 \AA$. ClustalX was used to combine the hTRIM5 $\alpha$ sequence with the structural alignments. A ClustalW sequence-based multiple alignment of 10 characteristic psiBLAST hits of all 3 sequences was used to localize insertions. A secondary structure prediction of hTRIM5 $\alpha$ obtained with Network Protein Sequence Analysis (NPS@; http://npsa-pbil.ibcp.fr) was used to manually correct the model. The final alignment was then fed into the model and loop optimization procedures of Modeller 8v2 (http://salilab.org/modeller), using $2 \mathrm{FBE}, 2 \mathrm{FNJ}$, and $2 \mathrm{IWG}$ as templates. 100 models were built and evaluated for stereochemical quality with PROCHECK. Pictures of the single best model were generated with PyMol v0.98. 
Immunofluorescence microscopy. CRFK cells stably expressing wild-type AoT5Cyp or hT5Cyp fusion proteins were grown on glass coverslips. Cells were fixed at $25^{\circ} \mathrm{C}$ for $10 \mathrm{~min}$ with $3.7 \%$ formaldehyde in PBS and permeabilized on ice for 2 min with $2 \%$ Triton X-100 in $0.1 \%$ sodium citrate. After quenching with $0.1 \mathrm{M}$ glycine in $\mathrm{PBS}$ at $25^{\circ} \mathrm{C}$ for 10 minutes, cells were blocked for 30 minutes at $25^{\circ} \mathrm{C}$ with $10 \% \mathrm{FBS}$ and $0.1 \%$ Tween-20 in PBS before overnight incubation with goat polyclonal anti-TRIM5 (AbCam) and mouse monoclonal anti-tubulin (Sigma-Aldrich) antibodies. After extensive washing in PBS, cells were sequentially incubated with donkey Alexa Fluor 488-anti-goat and goat Alexa Fluor 594-anti-mouse secondary antibodies (Invitrogen) for 1 hour at $25^{\circ} \mathrm{C}$ in the dark. Finally, coverslips were mounted in Vectashield with DAPI (Vector Laboratories). Cells were visualized with a $63 \times 1.4$ NA Leica HCX Planapochromat oil immersion objective using an inverted Leica DMI 6000 CS microscope fitted with a TCS SP5 laser-scanning confocal microscope system. Images from individual 3-dimensional stacks were subjected to noise reduction using the Leica LCS software (Leica Microsystems) and subjected to maximum intensity projection using the MetaMorph software (Universal Imaging Corp.).

Mice. Rag2 $2^{-/-\gamma c^{-/}}$mice on a BALB/c background (provided by M. Ito, Central Institute for Experimental Animals, Kawasaki, Japan) were bred and maintained under specific pathogen-free conditions in accordance with the guidelines of the animal facility at the Institute for Research in Biomedicine and the Swiss Federal Veterinary Office (Bern, Switzerland). Mouse experiments were reviewed and approved by the Veterinary Commission of the State of Ticino (Bellinzona, Switzerland). CL2MBP (clodronate) was a gift from Roche Diagnostics. Preparation of liposomes containing CL2MBP was done as described previously (68). Mice 6-10 weeks old received $100 \mu$ l of clodronate liposomes i.p. 24 hours prior to irradiation using a single sublethal dose of $4.0 \mathrm{~Gy}$ from a Cesium 137 source (Biobeam 8000; STS GmbH) at $3.75 \mathrm{~Gy} / \mathrm{min}$. Four hours after irradiation, mice were transplanted with $2.5 \times 10^{5} \mathrm{hCD} 4^{+} \mathrm{T}$ cells expressing hT5Cyp or control hT5CypH126Q in $100 \mu$ PBS i.p.. Mice were infected with HIV- $1_{\mathrm{NL} 4-3}$, as previously described (58), 5 or 14 days after transplant and analyzed 2,3 , or 4 weeks following infection. $\mathrm{CD}^{+} \mathrm{T}$ cell engraftment and maintenance in mice was measured by flow cytometry. To obtain peripheral blood cells and plasma, mice were bled from the retro-orbital venous sinus under anesthesia and red blood cells were lysed. When mice were sacrificed, single-cell suspensions from organs were prepared for FACS analysis and/or organs were assayed by immunohistochemistry. For FACS analysis, monoclonal antibodies against the following antigens were used: CD3 (UCHT1), CD4 (13B8.2), CD8 (B9.11), and p24 (clone KC57) (all Beckman Coulter) and CD45 (HI30) (Caltag). Intracellular staining for $\mathrm{p} 24$ was performed on single-cell suspensions from mesenteric lymph nodes and thymus following staining with anti-CD3 antibody. Cells were washed, permeabilized, and fixed by treatment with BD Cytofix/Cytoperm (BD Biosciences - Pharmingen) and stained with anti-p24 antibody in BD Permwash solution (BD Biosciences - Pharmingen) following the manufacturer's instructions. Plasma HIV RNA concentrations were determined by Cobas Amplicor RT-PCR assay (Roche Diagnostics). Immunohistochemical stainings were performed as previously described (58). To assess nonspecific binding, tissue from untransplanted and uninfected transplanted mice was stained as a control.

Statistics. Significant differences between groups were calculated using the Mann-Whitney $U$ test. $P$ values less than 0.05 were considered significant.

\section{Acknowledgments}

We thank David Jarrossay for cell sorting. This work was supported by NIH grants RO1AI36199 and RO1AI59159 to J. Luban, Swiss National Science Foundation grants 3100A0-113558 and 3100A0-102218 to J. Luban and M. Grütter, and funding from the Bill and Melinda Gates Foundation Grand Challenges in Global Health initiative, to M.G. Manz. M.R. Neagu was supported by the Columbia University College of Physicians and Surgeons Medical Scientist Training Program and the Roche Research Foundation.

Received for publication March 27, 2009, and accepted in revised form July 8, 2009.

Address correspondence to: Jeremy Luban, Department of Microbiology and Molecular Medicine, University of Geneva, 1 Rue Michel Servet, CH-1211 Geneva, Switzerland. Phone: 41-22-3795720; Fax: 41-22-379-5702; E-mail: jeremy.luban@unige.ch.
1. Fauci, A.S. 2008. 25 years of HIV. Nature. 453:289-290.

2. Barouch, D.H. 2008. Challenges in the development of an HIV-1 vaccine. Nature. 455:613-619.

3. Medzhitov, R., and Littman, D. 2008. HIV immunology needs a new direction. Nature. 455:591.

4. Strayer, D.S., et al. 2005. Current status of gene therapy strategies to treat HIV/AIDS. Mol. Ther. 11:823-842.

5. Baltimore, D. 2008. Presidential address. A global perspective on science and technology. Science. 322:544-551.

6. Stremlau, M., et al. 2004. The cytoplasmic body component TRIM5alpha restricts HIV-1 infection in Old World monkeys. Nature. 427:848-853.

7.Sayah, D.M., Sokolskaja, E., Berthoux, L., and Luban, J. 2004. Cyclophilin A retrotransposition into TRIM5 explains owl monkey resistance to HIV-1. Nature. 430:569-573.

8. Perez-Caballero, D., Hatziioannou, T., Zhang, F., Cowan, S., and Bieniasz, P.D. 2005. Restriction of human immunodeficiency virus type 1 by TRIMCypA occurs with rapid kinetics and independently of cytoplasmic bodies, ubiquitin, and proteasome activity. J. Virol. 79:15567-15572.

9. Diaz-Griffero, F., et al. 2006. Requirements for capsidbinding and an effector function in TRIMCyp-mediated restriction of HIV-1. Virology. 351:404-419.

10. Sebastian, S., and Luban, J. 2005. TRIM5alpha selectively binds a restriction-sensitive retroviral capsid. Retrovirology. 2:40

11. Stremlau, M., et al. 2006. Specific recognition and accelerated uncoating of retroviral capsids by the TRIM5alpha restriction factor. Proc. Natl. Acad. Sci. U. S. A. 103:5514-5519.

12. Luban, J. 2007. Cyclophilin A, TRIM5, and resistance to human immunodeficiency virus type 1 infection. J. Virol. 81:1054-1061.

13. Perez-Caballero, D., Hatziioannou, T., Yang, A., Cowan, S., and Bieniasz, P.D. 2005. Human tripartite motif 5alpha domains responsible for retrovirus restriction activity and specificity. J. Virol. 79:8969-8978

14. Stremlau, M., Perron, M., Welikala, S., and Sodroski, J. 2005. Species-specific variation in the B30.2(SPRY) domain of TRIM5alpha determines the potency of human immunodeficiency virus restriction. J. Virol. 79:3139-3145.

15. Sawyer, S.L., Wu, L.I., Emerman, M., and Malik, H.S. 2005. Positive selection of primate TRIM5alpha identifies a critical species-specific retroviral restriction domain. Proc. Natl. Acad. Sci. U. S. A. 102:2832-2837.

16. Yap, M.W., Nisole, S., and Stoye, J.P. 2005. A single amino acid change in the SPRY domain of human Trim5alpha leads to HIV-1 restriction. Curr. Biol. 15:73-78.

17. Yap, M.W., Dodding, M.P., and Stoye, J.P. 2006 Trim-cyclophilin A fusion proteins can restrict human immunodeficiency virus type 1 infection at two distinct phases in the viral life cycle. J. Virol. 80:4061-4067.

18. Schaller, T., Ylinen, L.M., Webb, B.L., Singh, S., and Towers, G.J. 2007. Fusion of cyclophilin a to $\mathrm{fv} 1$ enables cyclosporine-sensitive restriction of human and feline immunodeficiency viruses. J. Virol. 81:10055-10063.

19. Nisole, S., Lynch, C., Stoye, J.P., and Yap, M.W. 2004. A Trim5-cyclophilin A fusion protein found in owl monkey kidney cells can restrict HIV-1. Proc. Natl. Acad. Sci. U. S. A. 101:13324-13328.

20. Luban, J., Bossolt, K.L., Franke, E.K., Kalpana, G.V., and Goff, S.P. 1993. Human immunodeficiency virus type $1 \mathrm{Gag}$ protein binds to cyclophilins A and B. Cell. 73:1067-1078.

21. Ribeiro, I.P., et al. 2005. Evolution of cyclophilin $A$ and TRIMCyp retrotransposition in New World primates. J. Virol. 79:14998-15003.

22. Riddell, S.R., et al. 1996. T-cell mediated rejection of gene-modified HIV-specific cytotoxic T lymphocytes in HIV-infected patients. Nat. Med. 2:216-223.

23. Grutter, C., et al. 2006. Structure of the PRYSPRYdomain: implications for autoinflammatory diseases. FEBS Lett. 580:99-106.

24. Woo, J.S., et al. 2006. Structural and functional insights into the B30.2/SPRY domain. EMBO J. 25:1353-1363.

25. Ohkura, S., Yap, M.W., Sheldon, T., and Stoye, J.P. 2006. All three variable regions of the TRIM5alpha B30.2 domain can contribute to the specificity of 
retrovirus restriction. J. Virol. 80:8554-8565.

26. Sokolskaja, E., Berthoux, L., and Luban, J. 2006. Cyclophilin A and TRIM5alpha independently regulate human immunodeficiency virus type 1 infectivity in human cells. J. Virol. 80:2855-2862.

27. Braaten, D., Ansari, H., and Luban, J. 1997. The hydrophobic pocket of cyclophilin is the binding site for the human immunodeficiency virus type 1 Gag polyprotein. J. Virol. 71:2107-2113.

28. Chatterji, U., et al. 2005. Naturally occurring capsid substitutions render HIV-1 cyclophilin A independent in human cells and TRIM-cyclophilin-resistant in Owl monkey cells. J. Biol. Chem. 280:40293-40300.

29. Sebastian, S., Sokolskaja, E., and Luban, J. 2006. Arsenic counteracts human immunodeficiency virus type 1 restriction by various TRIM5 orthologues in a cell type-dependent manner. J. Virol. 80:2051-2054.

30. Saenz, D.T., Teo, W., Olsen, J.C., and Poeschla, E.M. 2005. Restriction of feline immunodeficiency virus by Ref1, Lv1, and primate TRIM5alpha proteins. J. Virol. 79:15175-15188.

31. Zhang, F., Hatziioannou, T., Perez-Caballero, D., Derse, D., and Bieniasz, P.D. 2006. Antiretroviral potential of human tripartite motif-5 and related proteins. Virology. 353:396-409.

32. Lin, T.Y., and Emerman, M. 2006. Cyclophilin A interacts with diverse lentiviral capsids. Retrovirology. 3:70.

33. Li, Y., Li, X., Stremlau, M., Lee, M., and Sodroski, J. 2006. Removal of arginine 332 allows human TRIM5alpha to bind human immunodeficiency virus capsids and to restrict infection. J. Virol. 80:6738-6744.

34. Anderson, J., and Akkina, R. 2008. Human immunodeficiency virus type 1 restriction by human-rhesus chimeric tripartite motif 5 alpha (TRIM 5alpha) in CD34(+) cell-derived macrophages in vitro and in $\mathrm{T}$ cells in vivo in severe combined immunodeficient (SCID-hu) mice transplanted with human fetal tissue. Hum. Gene Ther. 19:217-228.

35. Mazurier, F., et al. 1999. A novel immunodeficient mouse model--RAG2 x common cytokine receptor gamma chain double mutants--requiring exogenous cytokine administration for human hematopoietic stem cell engraftment. J. Interferon Cytokine Res. 19:533-541.

36. van Rijn, R.S., et al. 2003. A new xenograft model for graft-versus-host disease by intravenous transfer of human peripheral blood mononuclear cells in RAG2-/- gammac-/- double-mutant mice. Blood. 102:2522-2531.

37. Chen, B.K., Gandhi, R.T., and Baltimore, D. 1996. CD4 down-modulation during infection of human T cells with human immunodeficiency virus type 1 involves independent activities of vpu, env, and nef. J. Virol. 70:6044-6053.

38. Traggiai, E., et al. 2004. Development of a human adaptive immune system in cord blood cell-transplanted mice. Science. 304:104-107.

39. Liao, C.H., Kuang, Y.Q., Liu, H.L., Zheng, Y.T., and Su, B. 2007. A novel fusion gene, TRIM5-Cyclophilin A in the pig-tailed macaque determines its susceptibility to HIV-1 infection. AIDS. 21(Suppl 8):S19-S26.

40. Virgen, C.A., Kratovac, Z., Bieniasz, P.D., and Hatziioannou, T. 2008. Independent genesis of chimeric TRIM5-cyclophilin proteins in two primate species. Proc. Natl. Acad. Sci. U. S. A. 105:3563-3568.

41. Wilson, S.J., et al. 2008. Independent evolution of an antiviral TRIMCyp in rhesus macaques. Proc. Natl. Acad. Sci. U. S. A. 105:3557-3562.

42. Brennan, G., Kozyrev, Y., and Hu, S.L. 2008. TRIMCyp expression in Old World primates Macaca nemestrina and Macaca fascicularis. Proc. Natl. Acad. Sci. U. S. A. 105:3569-3574.

43. Newman, R.M., et al. 2008. Evolution of a TRIM5CypA splice isoform in old world monkeys. PLoS Pathog. 4:e1000003.

44. Song, B., et al. 2005. The B30.2(SPRY) domain of the retroviral restriction factor TRIM5alpha exhibits lineage-specific length and sequence variation in primates. J. Virol. 79:6111-6121.

45. Anderson, J., and Akkina, R. 2005. TRIM5alpharh expression restricts HIV-1 infection in lentiviral vector-transduced CD34+-cell-derived macrophages. Mol. Ther. 12:687-696.

46. Li, X., and Sodroski, J. 2008. The TRIM5alpha Bbox 2 domain promotes cooperative binding to the retroviral capsid by mediating higher-order selfassociation. J. Virol. 82:11495-11502.

47. Ranga, U., et al. 1998. Enhanced T cell engraftment after retroviral delivery of an antiviral gene in HIVinfected individuals. Proc. Natl. Acad. Sci. U. S. A. 95:1201-1206.

48. An, D.S., et al. 2007. Stable reduction of CCR5 by RNAi through hematopoietic stem cell transplant in non-human primates. Proc. Natl. Acad. Sci. U. S. A. 104:13110-13115.

49. Perez, E.E., et al. 2008. Establishment of HIV-1 resistance in CD4(+) $\mathrm{T}$ cells by genome editing using zinc-finger nucleases. Nat. Biotechnol. 26:808-816.

50. Kumar, P., et al. 2008. T cell-specific siRNA delivery suppresses HIV-1 infection in humanized mice. Cell. 134:577-586.

51. Boden, D., Pusch, O., and Ramratnam, B. 2007. Overcoming HIV-1 resistance to RNA interference. Front. Biosci. 12:3104-3116.

52. Hamm, T.E., Rekosh, D., and Hammarskjold, M.L. 1999. Selection and characterization of human immunodeficiency virus type 1 mutants that are resistant to inhibition by the transdominant negative RevM10 protein. J. Virol. 73:5741-5747.

53. Taylor, B.M., et al. 2008. An alteration of human immunodeficiency virus gp41 leads to reduced CCR5 dependence and CD4 independence. J. Virol. 82:5460-5471.
54. Glass, W.G., et al. 2006. CCR5 deficiency increases risk of symptomatic West Nile virus infection. J. Exp. Med. 203:35-40.

55. Berthoux, L., Sebastian, S., Sayah, D.M., and Luban, J. 2005. Disruption of human TRIM5alpha antiviral activity by nonhuman primate orthologues. J. Virol. 79:7883-7888.

56. Mosier, D.E., et al. 1991. Human immunodeficiency virus infection of human-PBL-SCID mice. Science. 251:791-794.

57. Namikawa, R., Kaneshima, H., Lieberman, M., Weissman, I.L., and McCune, J.M. 1988. Infection of the SCID-hu mouse by HIV-1. Science. 242:1684-1686.

58. Baenziger, S., et al. 2006. Disseminated and sustained HIV infection in CD34+ cord blood celltransplanted Rag2-/-gamma c-/- mice. Proc. Natl. Acad. Sci. U. S. A. 103:15951-15956.

59. Nakata, H., et al. 2005. Potent anti-R5 human immunodeficiency virus type 1 effects of a CCR5 antagonist, AK602/ONO4128/GW873140, in a novel human peripheral blood mononuclear cell nonobese diabetic-SCID, interleukin-2 receptor gamma-chain-knocked-out AIDS mouse model. J. Virol. 79:2087-2096.

60. Levine, B.L., et al. 2002. Adoptive transfer of costimulated CD4+ T cells induces expansion of peripheral T cells and decreased CCR5 expression in HIV infection. Nat. Med. 8:47-53.

61. Amendola, M., Venneri, M.A., Biffi, A., Vigna, E., and Naldini, L. 2005. Coordinate dual-gene transgenesis by lentiviral vectors carrying synthetic bidirectional promoters. Nat. Biotechnol. 23:108-116.

62. Goldstein, H. 2008. Summary of presentations at the NIH/NIAID New Humanized Rodent Models 2007 Workshop. AIDS Res. Ther. 5:3.

63. Manz, M.G. 2007. Human-hemato-lymphoid-system mice: opportunities and challenges. Immunity. 26:537-541.

64. Berthoux, L., Sebastian, S., Sokolskaja, E., and Luban, J. 2004. Lv1 inhibition of human immunodeficiency virus type 1 is counteracted by factors that stimulate synthesis or nuclear translocation of viral cDNA. J. Virol. 78:11739-11750.

65. Papkalla, A., Munch, J., Otto, C., and Kirchhoff, F. 2002. Nef enhances human immunodeficiency virus type 1 infectivity and replication independently of viral coreceptor tropism. J. Virol. 76:8455-8459.

66. Lois, C., Hong, E.J., Pease, S., Brown, E.J., and Baltimore, D. 2002. Germline transmission and tissue-specific expression of transgenes delivered by lentiviral vectors. Science. 295:868-872.

67. Mangeot, P.E., et al. 2002. High levels of transduction of human dendritic cells with optimized SIV vectors. Mol. Ther. 5:283-290.

68. Van Rooijen, N., and Sanders, A. 1994. Liposome mediated depletion of macrophages: mechanism of action, preparation of liposomes and applications. J. Immunol. Methods. 174:83-93. 